\title{
PRIMITIVE DU COCYCLE DE MASLOV GÉNÉRALISÉ
}

\author{
JEAN-LOUIS CLERC \& KHALID KOUFANY
}

\begin{abstract}
RÉsumé. Soit $\mathcal{D}$ un espace hermitien symétrique de type tube, de frontière de Shilov $S$. Nous décrivons une réalisation du revêtement universel $\widetilde{S}$ de $S$. Nous construisons ensuite sur $\widetilde{S}$ une primitive du cocycle de Maslov généralisé tel que défini dans [Transform. Groups 6 (2001), 303-320] et [J. Math. Pures Appl. 83 (2004), 99-114]. C'est l'analogue de l'indice de Souriau pour la variété lagrangienne. Une variante de cette construction généralise l'indice d'ArnoldLeray-Maslov. Enfin, nous utilisons cette primitive pour généraliser la notion de nombre de rotation symplectique.
\end{abstract}

\section{INTRODUCTION}

Soit $(E, \omega)$ un espace symplectique réel, de dimension $2 r$. Un lagrangien est un sous-espace totalement isotrope (pour la forme $\omega$ ) de dimension maximale (égale à $r$ ). L'ensemble des lagrangiens de $E$, noté $S$, est naturellement muni d'une structure de variété compacte et est appelé la variété lagrangienne. Le groupe symplectique $G=S p(E)$ opère transitivement sur $S$.

A un triplet de lagrangiens $\left(l_{1}, l_{2}, l_{3}\right)$, on associe son indice de Maslov $\iota\left(l_{1}, l_{2}, l_{3}\right)$, dont une définition algébrique a été proposée par Kashiwara (voir $[\mathrm{L}-\mathrm{V}]$ ). Les principales propriétés de l'indice de Maslov sont les suivantes :

(i) l'indice de Maslov est invariant par $G$, c'est-à-dire :

$$
\iota\left(g l_{1}, g l_{2}, g l_{3}\right)=\iota\left(l_{1}, l_{2}, l_{3}\right)
$$

pour tout $g \in G$ et $l_{1}, l_{2}, l_{3} \in S$.

(ii) l'indice de Maslov est antisymétrique pour les permutations des trois arguments, c'est-à-dire :

$$
\iota\left(l_{\tau(1)}, l_{\tau(2)}, l_{\tau(3)}\right)=\epsilon(\tau) \iota\left(l_{1}, l_{2}, l_{3}\right)
$$

où $\epsilon(\tau)$ désigne la signature de la permutation $\tau$.

(iii) l'indice de Maslov satisfait une relation de cocycle

$$
\iota\left(l_{1}, l_{2}, l_{3}\right)=\iota\left(l_{1}, l_{2}, l_{4}\right)+\iota\left(l_{2}, l_{3}, l_{4}\right)+\iota\left(l_{3}, l_{1}, l_{4}\right)
$$

pour tous $l_{1}, l_{2}, l_{3}, l_{4} \in S$.

La variété lagrangienne est un cas particulier d'une famille d'espaces, liée aux algèbres de Jordan euclidiennes. On renvoie à $[\mathrm{F}-\mathrm{K}]$ pour les définitions, notations et résultats concernant ces algèbres.

1991 Mathematics Subject Classification. 32M15; 53D12; 15A66.

Key words and phrases. Espace hermitien symétrique de type tube ; Frontière de Shilov ; Indice d'Arnold-Leray-Maslov; Indice d'inertie; Indice de Maslov; Indice de Souriau. 
Selon un premier point de vue, on voit $S$ comme la frontière de Shilov d'un domaine de type tube. Plus précisément, soit $J$ une algèbre de Jordan euclidienne, $\Omega$ son cône (ouvert, propre, convexe, homogène, auto-adjoint) des carrés inversibles. Notons J l'algèbre de Jordan complexifiée de $J$. Elle est naturellement munie d'une norme spectrale. La boule unité ouverte correspondante

$$
\mathcal{D}=\{z \in \mathbb{J},|z|<1\}
$$

munie de la métrique de Bergmann, est un domaine hermitien symétrique sous l'action du groupe $G$ des difféomorphismes holomorphes de $\mathcal{D}$. Par une transformée de Cayley, le domaine $\mathcal{D}$ est holomorphiquement équivalent au tube

$$
T_{\Omega}=J+i \Omega
$$

d'où le nom de domaine de type tube. On désigne par $S$ la frontière de Shilov de $\mathcal{D}$. Lorsque $J$ est l'algèbre $\operatorname{Sym}(r, \mathbb{R})$ des matrices $r \times r$ réelles symétriques pour le produit $x \cdot y=\frac{1}{2}(x y+y x)$, le domaine $\mathcal{D}$ correspondant est le disque de Siegel, le groupe $G$ est essentiellement le groupe symplectique et la frontière de Shilov s'identifie à la variété lagrangienne évoquée ci-dessus.

Il existe un deuxième point de vue, qui ne fait pas appel à la théorie des domaines hermitiens symétriques. La variété $S$ peut être obtenue comme complétée conforme de l'algèbre de Jordan $J$, le groupe $G$ apparaissant comme le groupe des transformations conformes de $J$ suivant la terminologie de [B]. Une variante de ce point de vue consiste à munir la variété $S$ d'une structure causale, et $G$ est alors le groupe des transformations causales de $S$.

Dans $\left[\mathrm{C}_{2}\right]$, achevant le programme commencé dans $\left[\mathrm{C}-\varnothing_{1}\right]$ et $\left[\mathrm{C}-\varnothing_{2}\right]$, l'un des deux présents auteurs a proposé une généralisation de l'indice de Maslov pour l'action de $G$ sur $S \times S \times S$. L'approche, nouvelle même pour le cas de la variété lagrangienne, utilise principalement la réalisation de $S$ comme frontière de Shilov du domaine $\mathcal{D}$. L'objectif du présent article est de faire le lien avec les constructions classiques dues à Maslov, Arnold, Souriau et Leray (voir $[\mathrm{M}],\left[\mathrm{A}_{1}\right],\left[\mathrm{A}_{2}\right],[\mathrm{S}]$, [Le], $\left.\left[\mathrm{G}_{2}\right]\right)$. Plus précisément, l'indice de Maslov satisfait une relation de cocycle. Lorsqu'on passe au revêtement universel $\widetilde{S}$ de $S$, on cherche une primitive de ce cocycle, c'est-à-dire une fonction $m: \widetilde{S} \times \widetilde{S} \longrightarrow \mathbb{Z}$, antisymétrique et telle que

$$
\iota\left(\sigma_{1}, \sigma_{2}, \sigma_{3}\right)=m\left(\widetilde{\sigma}_{1}, \widetilde{\sigma}_{2}\right)+m\left(\widetilde{\sigma}_{2}, \widetilde{\sigma}_{3}\right)+m\left(\widetilde{\sigma}_{3}, \widetilde{\sigma}_{1}\right)
$$

pour tout triplet $\left(\widetilde{\sigma}_{1}, \widetilde{\sigma}_{2}, \widetilde{\sigma}_{3}\right)$ de points de $\widetilde{S}$, de projections respectives $\sigma_{1}, \sigma_{2}, \sigma_{3}$ sur $S$. Dans une variante, on introduit l'indice d'inertie d'un triplet de points de $S$. Celui-ci possède encore la propriété d'invariance par le groupe $G$ et une propriété de cocycle (mais n'a pas la propriété d'antisymétrie), et on se propose également d'en chercher une primitive.

Les deux constructions, quoique essentiellement inspirées par les travaux antérieurs, en diffèrent sur quelques points. L'utilisation de la structure causale sur $S$ et la description d'un revêtement $\Gamma$ du groupe $G$ qui opère sur $\widetilde{S}$ sont nouvelles, même dans le cas classique, et permettent notamment de donner des définitions dont l'invariance par $\Gamma$ est manifeste.

Le plan de l'article est le suivant.

Le paragraphe 2 présente les domaines de type tube et leurs frontières de Shilov, du point de vue des algèbres de Jordan euclidiennes. La structure causale sur $S$ y 
est également introduite. Au paragraphe 3, on donne une réalisation du revêtement universel $\widetilde{S}$ de $S$, puis on construit un revêtement $\Gamma$ du groupe $G$ qui opère sur $\widetilde{S}$. Au paragraphe 4 , on étudie l'action de $G$ sur $S \times S$, et on précise un invariant caractéristique pour les couples de points de $S$, appelé indice de transversalité.

Après ce travail préparatoire, on commence au paragraphe 5 la construction de l'indice de Souriau dans le cas transverse, étendu au cas non transverse au paragraphe 6, en suivant une idée de de Gosson (voir $\left[\mathrm{G}_{1}\right]$ ).

Au paragraphe 7 , on introduit le cycle de Maslov et on établit ses propriétés géométriques. Elles permettent au paragraphe 8 de définir le nombre d'Arnold (directement sans hypothèse de transversalité). On construit ensuite l'indice d'ArnoldLeray-Maslov, qui fournit une primitive de l'indice d'inertie (défini par modification de l'indice de Maslov).

Au paragraphe 9, on montre, sans s'appesantir sur les détails, que le concept de l'indice de Maslov de deux chemins tel que développé dans [C-L-M] peut aussi se généraliser dans notre contexte. Enfin, au paragraphe 10, on construit pour le groupe $G$ l'analogue du nombre de rotation symplectique introduit dans [B-G].

\section{La frontière De Shilov D'Un ESPaCe hermitien SymÉtrique DE Type} TUBE

Soit $J$ une algèbre de Jordan euclidienne simple d'élément unité $e$, de dimension $n$ et de rang $r$. Son invariant de Peirce $d$ est tel que

$$
n=r+\frac{r(r-1)}{2} d .
$$

Soit det le déterminant de $J$. C'est une fonction polynomiale homogène de degré $r$. La trace de $J$, notée $\operatorname{tr}$ est une forme linéaire sur $J$. On peut supposer que le produit salaire de $J$ est défini par

$$
\langle x \mid y\rangle=\operatorname{tr}(x y) .
$$

Par complexification on obtient une algèbre de Jordan complexe $\mathbb{J}$. La conjugaison par rapport à la forme réelle $J$ est notée $\eta$, mais on utilisera aussi la notation

$$
\eta(z)=\bar{z} .
$$

Les extensions holomorphes de det et tr à $\mathbb{I}$ seront encore notées det et tr respectivement.

Le produit scalaire (1) s'étend en un produit hermitien sur $\mathbb{J}$ donné par

$$
\langle z \mid w\rangle=\operatorname{tr}(z \bar{w}) .
$$

Soit

$$
S=\left\{\sigma \in \mathbb{J} \mid \bar{\sigma}=\sigma^{-1}\right\} .
$$

C'est une sous-variété compacte connexe de J. Nous allons résumer certaines de ses caractérisations dans la proposition suivante (voir [F-K, Proposition X.2.3]) :

Proposition 2.1. Pour $\sigma \in \mathbb{J}$, les assertions suivantes sont équivalentes :

(i) $\sigma \in S$,

(ii) $\sigma=\exp ($ iu) où $u \in J$,

(iii) il existe un repère de Jordan $\left(c_{j}\right)_{1 \leq j \leq r}$ de $J$ et des nombres complexes $\zeta_{1}, \ldots, \zeta_{r}$ de module 1 , tels que $\sigma=\sum_{j=1}^{\bar{r}} \zeta_{j} c_{j}$. 
On note $\mathbb{L}=S \operatorname{tr}(\mathbb{J})$ le groupe de structure de l'algèbre de Jordan complexe $\mathbb{J}$. Considérons

$$
L(S)=\{g \in G L(\mathbb{J}) \mid g(S)=S\} .
$$

Alors $L(S)=\mathbb{L} \cap U(\mathbb{J})$, où $U(\mathbb{J})$ désigne le groupe unitaire de $\mathbb{J}$ muni de la forme hermitienne (3). Ce groupe opère transitivement sur $S$. Le stabilisateur de $e$ dans $L(S)$ cö̈ncide avec le groupe $A u t(J)$ des automorphismes de l'algèbre de Jordan $J$ (on étend l'action des éléments de $A u t(J)$ de manière $\mathbb{C}$-linéaire à $\mathbb{J}$ ). L'involution $\eta$ introduite en (2) conserve $S$, et admet $e$ comme point fixe isolé. L'involution correspondante de $L(S)$ donnée par $g \mapsto \eta \circ g \circ \eta$ a pour points fixes exactement $L(S)_{e}=A u t(J)$. En d'autres termes, muni de la métrique induite par le produit hermitien (3), $S$ est un espace riemannien symétrique compact, isomorphe à $L(S) / A u t(J)$.

Comme $L(S)$ n'est pas nécessairement connexe, on préfère introduire sa composante connexe neutre $U$, qui est encore transitive sur $S$, et on désigne par $U_{e}$ le stabilisateur dans $U$ de l'élément $e$. On a $\operatorname{Aut}(J)^{\circ} \subset U_{e} \subset \operatorname{Aut}(J)$ et

$$
S \simeq U / U_{e} .
$$

Soit $\left(c_{j}\right)_{1 \leq j \leq r}$ un repère de Jordan. Pour tout élément $z \in \mathbb{J}$, il existe $u \in U$ et il existe des scalaires $0 \leq \lambda_{1} \leq \ldots \leq \lambda_{r}$ uniques tels que

$$
z=u\left(\sum_{j=1}^{r} \lambda_{j} c_{j}\right) .
$$

La norme spectrale de $z$ est définie par

$$
|z|=\sup \lambda_{j}
$$

et la fonction $z \mapsto|z|$ est une norme sur $\mathbb{J}$ invariante par $U$. Soit $\mathcal{D}$ la boule unité ouverte de $\mathbb{J}$ pour la norme spectrale

$$
\mathcal{D}=\{z \in \mathbb{J},|z|<1\} .
$$

Théorème 2.2 (voir $[\mathrm{F}-\mathrm{K}$, Theorem X.4.6]). D est un domaine borné hermitien de type tube dont la frontière de Shilov est $S$.

Le domaine $\mathcal{D}$ peut être réalisé comme un domaine tube au dessus d'un cône ouvert de $J$. Plus précisément, l'ensemble des carrés de $J$ est un cône convexe fermé et son intérieur $\Omega$ est un cône ouvert, convexe, propre, et auto-dual. C'est le cône symétrique associé à $J$.

Le groupe $L(\Omega)$ des transformations linéaires de $J$ qui préservent $\Omega$ est un groupe de Lie réductif qui agit transitivement sur $\Omega$. Soit $L$ sa composante connexe neutre. Le stabilisateur $K$ de l'identité $e$ dans $L$ est un sous-groupe compact maximal de $L$. C'est la composante connexe neutre du groupe $A u t(J)$ et on a $K=L \cap O(J)$, où $O(J)$ est le groupe orthogonal de $J$.

Soit $T_{\Omega}$ le domaine tube dans $\mathbb{J}$ de base $\Omega$

$$
T_{\Omega}=J+i \Omega=\{z=x+i y \mid x \in J, y \in \Omega\} .
$$

La transformation de Cayley $c$ et son inverse $p$ sont données par

$$
\begin{aligned}
& p(z)=(z-i e)(z+i e)^{-1}=e-2 i(z+i e)^{-1}, \\
& c(w)=i(e+w)(e-w)^{-1}=-i e+2 i(e-w)^{-1},
\end{aligned}
$$

et on a (voir [F-K, Proposition X.2.3, Theorem X.4.3]) : 
Théorème 2.3. L'application $p$ induit un isomorphisme biholomorphe de $T_{\Omega}$ sur $\mathcal{D}$, et

$$
p(J)=\{\sigma \in S \mid \operatorname{det}(e-\sigma) \neq 0\} .
$$

Les deux domaines $\mathcal{D}$ et $T_{\Omega}$ sont holomorphiquement équivalents. $T_{\Omega}$ est la réalisation non bornée de $\mathcal{D}$ et on peut voir $J$ comme sa frontière de Shilov; son image par $p$ est un ouvert dense dans $S$.

Soit $G$ la composante connexe neutre du groupe des automorphismes holomorphes de $\mathcal{D}$. C'est un groupe de Lie semi-simple et le stabilisateur de 0 dans $G$ est un sous-groupe compact maximal de $G$ et coïncide avec le groupe $U$.

Soit $G\left(T_{\Omega}\right)$ la composante connexe neutre du groupe des automorphismes holomorphes de $T_{\Omega}$. Les éléments du groupe $L$ opèrent sur $T_{\Omega}$ par extension $\mathbb{C}$-linéaire et on peut considérer $L$ comme un sous-groupe de $G\left(T_{\Omega}\right)$. En fait $G\left(T_{\Omega}\right)$ est engendré par $L$, le sous-groupe abélien $N^{+}$des translations

$$
t_{u}: z \mapsto z+u, \quad u \in J
$$

et par l'inversion

$$
s: z \mapsto-z^{-1} .
$$

Le sous-groupe abélien $N^{-}=s \circ N^{+} \circ s$ est l'ensemble des transformations

$$
\widetilde{t}_{u}=s \circ t_{u} \circ s: z \mapsto\left(z^{-1}+u\right)^{-1}, u \in J .
$$

On a

$$
G\left(T_{\Omega}\right)=c \circ G \circ c^{-1} .
$$

Le produit semi-direct $P=L \ltimes N^{-}$est un sous-groupe parabolique maximal de $G\left(T_{\Omega}\right)$. L'espace homogène $G\left(T_{\Omega}\right) / P$ est donc une variété (réelle) compacte contenant $J$ comme un sous-ensemble ouvert dense, le plongement de $J$ dans $G\left(T_{\Omega}\right) / P$ étant donné par

$$
J \rightarrow G\left(T_{\Omega}\right) / P: u \mapsto t_{u} P .
$$

$G\left(T_{\Omega}\right) / P$ est la compactification conforme de $J$ (voir [B]), elle est isomorphe de la frontière de Shilov $S$ de $\mathcal{D}$.

Donnons enfin un troisième point de vue sur la géométrie de $S$, à savoir l'existence d'une structure causale $G$-invariante sur $S$.

Un cône causal d'un espace vectoriel de dimension finie est un cône non vide $C$, convexe, ouvert et d'adhérence pointue (c'est-à-dire $\bar{C} \cap-\bar{C}=\{0\}$ ). Une structure causale sur une variété $\mathcal{M}$ est la donnée d'un champs de cônes $\left(C_{p}\right)_{p \in \mathcal{M}}$ sur $\mathcal{M}$ tel que, pour tout $p \in \mathcal{M}, C_{p}$ est un cône causal dans l'espace tangent $T_{p}(\mathcal{M})$, qui dépend différentiablement de $p$. Une courbe $\gamma:[a, b] \rightarrow \mathcal{M}$ de classe $\mathcal{C}^{1}$ est une courbe causale (resp. anti-causale) si, pour tout $t, \dot{\gamma}(t) \in C_{\gamma(t)}\left(\operatorname{resp} . \dot{\gamma}(t) \in-C_{\gamma(t)}\right)$. Lorsque $\mathcal{M}$ est un espace homogène sous l'action d'un groupe de Lie $G$, la structure causale est dite $G$-invariante si $C_{g(x)}=D g(x)\left(C_{x}\right)$, pour tout $g \in G$ et tout $x \in \mathcal{M}$. Soit $x_{\circ}$ un point base de $\mathcal{M}$. Une structure causale $G$-invariante sur $\mathcal{M}$ est complètement déterminée par la donnée d'un cône causal $C$, dans $T_{x_{\diamond}}(\mathcal{M})$, invariant par l'action du stabilisateur de $x_{\circ}$ dans $G$.

L'algèbre de Jordan $J$ admet une structure causale naturelle, modelée par le cône symétrique $\Omega$. Il s'agit de la donnée en chaque point de $J$ du cône $\Omega$, vu comme un 
cône dans l'espace tangent à $J$ en ce point. Cette structure causale est invariante $\operatorname{par} G\left(T_{\Omega}\right)$.

La transformation de Cayley permet de transporter à $S$ cette structure causale. Considérons $-e \in S$ comme point base de $S$, alors l'espace tangent à $S$ en $-e$ s'identifie à $i J$. Par ailleurs, $p(0)=-e$, et la transformation de Cayley $p=c^{-1}$ est bien définie dans un voisinage de 0 . Sa différentielle en 0 est

$$
D p(0)=-2 i \operatorname{Id}_{\mathbb{J}} .
$$

On définit alors un cône causal en $-e$ en posant

$$
C_{-e}=\operatorname{Dp}(0)(\Omega)=-i \Omega \text {. }
$$

Comme ce cône est invariant par le stabilisateur du point $-e$ dans $G$, on peut définir une structure causale sur $S$ invariante par $G$ de la manière suivante : si $\sigma$ est un point quelconque de $S$, alors il existe $g \in G$ tel que $g(-e)=\sigma$. Le cône

$$
C_{\sigma}=D g(-e)\left(C_{-e}\right),
$$

est un cône causal contenu dans l'espace tangent à $S$ en $\sigma$, ne dépendant pas du choix de $g$. Nous obtenons ainsi un champs de cônes causaux $\left(C_{\sigma}\right)_{\sigma \in S}$ invariants par $G$. Enfin, il est clair que le cône causal $C_{\sigma}$ dépend différentiablement de $\sigma$.

Pour finir ce paragraphe, nous donnons la liste des algèbres de Jordan euclidiennes, les domaines hermitiens bornés correspondants et leurs frontières de Shilov.

\begin{tabular}{cccc}
\hline$J$ & $\mathbb{J}$ & $\mathcal{D}$ & $S$ \\
\hline $\operatorname{Sym}(m, \mathbb{R})$ & $\operatorname{Sym}(m, \mathbb{C})$ & $S p(2 m, \mathbb{R}) / U(m)$ & $U(m) / O(m)$ \\
$\operatorname{Herm}(m, \mathbb{C})$ & $\operatorname{Mat}(m, \mathbb{C})$ & $S U(m, m) / S(U(m) \times U(m))$ & $U(m)$ \\
$\operatorname{Herm}(m, \mathbb{H})$ & $\operatorname{Skew}(2 m, \mathbb{C})$ & $S O^{*}(4 m) / U(2 m)$ & $U(2 m) / S U(m, \mathbb{H})$ \\
$\mathbb{R} \times \mathbb{R}^{q-1}$ & $\mathbb{C} \times \mathbb{C}^{q-1}$ & $S O_{0}(2, q) / S O(2) \times S O(q)$ & $\left(U(1) \times S^{q-1}\right) / \mathbb{Z}_{2}$ \\
$\operatorname{Herm}(3, \mathbb{O})$ & $\operatorname{Mat}(3, \mathbb{O})$ & $E_{7(-25)} / U(1) E_{6}$ & $U(1) E_{6} / F_{4}$ \\
\hline
\end{tabular}

TAB. 1 - Domaines hermitiens bornés et leurs frontières de Shilov

\begin{tabular}{cccc}
\hline$J$ & $n$ & $r$ & $d$ \\
\hline $\operatorname{Sym}(m, \mathbb{R})$ & $\frac{1}{2} m(m+1)$ & $m$ & 1 \\
$\operatorname{Herm}(m, \mathbb{C})$ & $m^{2}$ & $m$ & 2 \\
$\operatorname{Herm}(m, \mathbb{H})$ & $m(2 m-1)$ & $m$ & 4 \\
$\mathbb{R} \times \mathbb{R}^{q-1}$ & $q$ & 2 & $q-2$ \\
$\operatorname{Herm}(3, \mathbb{O})$ & 27 & 3 & 8 \\
\hline
\end{tabular}

TAB. 2 - La dimension, le rang et l'invariant de Peirce de $J$

\section{Le Revêtement universel De la frontière De Shilov}

La variété $S$ vue comme espace riemannien symétrique $\simeq U / U_{e}$ n'est pas semisimple mais seulement réductif. En effet, $S$ est stable par la multiplication par un nombre complexe $e^{i \theta}$ de module 1 , et ceci montre qu'il y a un sous-groupe isomorphe 
au groupe des nombres complexes de module 1 dans le centre de $U$. Considérons maintenant

$$
S_{1}=\{\sigma \in S \mid \operatorname{det}(\sigma)=1\}
$$

Proposition 3.1. $S_{1}$ est une sous-variété connexe de S. C'est un espace riemannien symétrique semi-simple de type compact.

Démonstration. Près du point $e \in S$, on peut utiliser la carte locale de $S$ donnée par

$$
J \ni x \longmapsto \exp i x \in S .
$$

La condition $\sigma \in S_{1}$ se traduit par $\operatorname{tr}(x)=0$, ce qui montre bien que $S_{1}$ est une sous-variété près de $e$. On utilise alors l'action du groupe $U$ pour en déduire que $S_{1}$ est (globalement) une sous-variété de $S$. Pour la connexité, on va montrer que $S_{1}=\exp i J_{0}$, où

$$
J_{0}=\{v \in J \mid \operatorname{tr}(v)=0\} .
$$

Comme $\operatorname{det}(\exp i v)=e^{i \operatorname{tr}(v)}=1$ pour tout $v \in J_{0}$, seule l'inclusion $S_{1} \subset \exp i J_{0}$ est à démontrer. Soit donc $\sigma \in S_{1}$. D'après la proposition 2.1 on a $S=\exp i J$, il existe donc $v \in J$ tel que $\exp i v=\sigma$. Comme

$$
1=\operatorname{det}(\sigma)=\operatorname{det}(\exp i v)=e^{i \operatorname{tr}(v)},
$$

on a $\operatorname{tr}(v)=2 k \pi$ avec $k \in \mathbb{Z}$. Soit $\left(c_{j}\right)_{1 \leq j \leq r}$ un repère de Jordan adapté à $v$, c'est-à-dire tel que $v=\sum_{j=1}^{r} a_{j} c_{j}$, où $a_{j} \in \mathbb{R}$ pour tout $j$. Soit

$$
w=v-2 k \pi c_{1}=\left(a_{1}-2 k \pi\right) c_{1}+\sum_{j=2}^{r} a_{j} c_{j}
$$

Alors

$$
\operatorname{tr}(w)=\operatorname{tr}(v)-2 k \pi \operatorname{tr}\left(c_{1}\right)=0,
$$

car $\operatorname{tr}(c)=1$ pour tout idempotent primitif $c$, et

$$
\exp w=e^{i\left(a_{1}-2 k \pi\right)} c_{1}+\sum_{j=2}^{r} e^{i a_{j}} c_{j}=\sum_{j=1}^{r} e^{i a_{j}} c_{j}=\exp v=\sigma .
$$

Donc $\sigma \in \exp i J_{0}$ et par suite $S_{1}=\exp i J_{0}$, ce qui montre que $S_{1}$ est connexe.

Le polynôme det est relativement invariant par l'action du groupe de structure $\mathbb{L}$ de $\mathbb{J}$, et il y a donc un caractère $\chi$ de $\mathbb{L}$ à valeurs dans $\mathbb{C}^{*}$ tel que

$$
\operatorname{det}(g z)=\chi(g) \operatorname{det}(z)
$$

pour tout $g \in \mathbb{L}$ et tout $z \in \mathbb{J}$. Soit

$$
U_{1}=\{u \in U \mid \chi(u)=1\} .
$$

Le sous-groupe $U_{1}$ opère sur $S_{1}$, et cette action est transitive. En effet, si $\sigma \in S_{1}$, il existe $u \in U$ tel que $\sigma=u(e)$. Alors

$$
1=\operatorname{det}(\sigma)=\chi(u) \operatorname{det}(e)=\chi(u),
$$

et donc $u \in U_{1}$.

La composante connexe neutre $U_{1}^{\circ}$ de $U_{1}$ opère donc aussi transitivement sur $S_{1}$. De plus, si $u \in U$ est un élément qui fixe $e$, alors $\chi(u)=1$ et donc $U_{e} \subset U_{1}$. En particulier, $\left(U_{1}\right)_{e}$ est le sous-groupe des points fixes de l'involution $g \mapsto \eta \circ g \circ \eta$ (où $\eta$ est donnée par (2)), ce qui réalise $S_{1}$ comme espace symétrique

$$
S_{1}=U_{1}^{\circ} /\left(U_{1}^{\circ} \cap \operatorname{Aut}(J)\right) \text {. }
$$


Reste à voir que $U_{1}^{\circ}$ est semi-simple. L'algèbre de Lie de $U$ est $\mathfrak{u}=\mathfrak{k} \oplus i \mathfrak{p}$, où $\mathfrak{k}=\operatorname{Der}(J)$ est l'algèbre des dérivations de l'algèbre de Jordan $J$, et

$$
\mathfrak{p}=\{L(v) \mid v \in J\},
$$

où $L(v)$ désigne l'opérateur de multiplication par $v$ dans $J$. Donc clairement

$$
\mathfrak{u}_{1}=\operatorname{Lie}\left(U_{1}\right)=\mathfrak{k} \oplus i \mathfrak{p}_{0}
$$

où

$$
\mathfrak{p}_{0}=\{L(v) \mid v \in J, \operatorname{tr}(v)=0\}=\left\{L(v) \mid v \in J_{0}\right\} .
$$

Le centre de $\mathfrak{u}$ est $\mathbb{R} L(e)=\mathbb{R I d}$, de sorte que le centre de $\mathfrak{u}_{1}$ est réduit à $\{0\}$, et donc $\mathfrak{u}_{1}$ est bien semi-simple.

Pour décider si un espace symétrique semi-simple de type compact $U / K$ est simplement connexe (ou plus généralement pour calculer son groupe fondamental), rappelons qu'il faut comparer deux réseaux dans un sous-espace de Cartan : l'un , noté $\Lambda_{0}$ est obtenu algébriquement à partir de données radicielles, l'autre $\Lambda$ est obtenue à partir de la détermination des éléments $H$ d'un sous-espace de Cartan tels que $\exp H \in K$.

Fixons un repère de Jordan $\left(c_{j}\right)_{1 \leq j \leq r}$, et soit

$$
\mathfrak{a}=\left\{L(a) \mid a=\sum_{j=1}^{r} a_{j} c_{j}, a_{j} \in \mathbb{R}\right\} .
$$

C'est un sous-espace de Cartan de $\mathfrak{p}$, et par suite

$$
\mathfrak{a}_{0}=\left\{L(a) \mid a=\sum_{j=1}^{r} a_{j} c_{j}, a_{j} \in \mathbb{R}, \operatorname{tr}(a)=0\right\} .
$$

est un sous-espace de Cartan de $\mathfrak{p}_{0}$. On munit $\mathfrak{p}$ (et donc $\mathfrak{a}$ ) du produit scalaire défini par

$$
(L(x) \mid L(y))=\langle x \mid y\rangle,
$$

pour $x, y \in J$. Il est proportionnel au produit scalaire induit par la forme de Killing de $\mathfrak{u}$.

Le système des racines restreintes de $(\mathfrak{u}, \mathfrak{a})$ est

$$
\Sigma(\mathfrak{u}, \mathfrak{a})=\left\{\frac{1}{2}\left(a_{j}-a_{k}\right), 1 \leq j \neq k \leq r\right\},
$$

(voir $\left[\mathrm{F}-\mathrm{K}\right.$, page 212]) et c'est aussi le système des racines restreintes de $\left(\mathfrak{u}_{0}, \mathfrak{a}_{0}\right)$. Considérons les deux réseaux de $\mathfrak{a}_{0}$ :

$$
\Lambda_{0}=\text { réseau engendré par }\left\{2 \pi \frac{A_{j, k}}{\left(A_{j, k} \mid A_{j, k}\right)} \mid 1 \leq j \neq k \leq r\right\} \text {, }
$$

où $A_{j, k}$ est le covecteur de $\frac{1}{2}\left(a_{j}-a_{k}\right)$, et

$$
\Lambda=\left\{H \in \mathfrak{a}_{0} \mid \exp (i H) e=e\right\} .
$$

Lemme 3.2. Le réseau $\Lambda_{0}$ est engendré par les éléments

$$
2 \pi L\left(c_{j}-c_{k}\right), \quad 1 \leq j \neq k \leq r .
$$


Démonstration. En effet, l'élément $A_{j, k}=\frac{1}{2} L\left(c_{j}-c_{k}\right) \in \mathfrak{a}_{0}$ est le covecteur ${ }^{1}$ de la racine $\frac{1}{2}\left(a_{j}-a_{k}\right)$ en ce sens que pour $a=\sum_{j=1}^{r} a_{j} c_{j}$,

$$
\left\langle\frac{1}{2}\left(c_{j}-c_{k}\right) \mid a\right\rangle=\frac{1}{2}\left(a_{j}-a_{k}\right) .
$$

Notons par ailleurs que

$$
\left(A_{j, k} \mid A_{j, k}\right)=\left\langle\frac{1}{2}\left(c_{j}-c_{k}\right) \mid \frac{1}{2}\left(c_{j}-c_{k}\right)\right\rangle=\frac{1}{2} .
$$

D'où

$$
2 \pi \frac{A_{j, k}}{\left(A_{j, k} \mid A_{j, k}\right)}=2 \pi L\left(c_{j}-c_{k}\right)
$$

Lemme 3.3. On a

$$
\Lambda=\left\{L(a) \mid a=\sum_{j=1}^{r} 2 \pi m_{j} c_{j}, m_{j} \in \mathbb{Z}, \sum_{j=1}^{r} m_{j}=0\right\} .
$$

Démonstration. Soit $a=\sum_{j=1}^{r} a_{j} c_{j}$. On a

$$
\exp i L(a)=P\left(\exp \left(\frac{i a}{2}\right)\right)
$$

où $P$ désigne l'application quadratique de l'algèbre de Jordan $J$, et

$$
P\left(\exp \left(\frac{i a}{2}\right)\right) e=\exp (i a)
$$

Donc

$$
L(a) \in \Lambda \Longleftrightarrow \exp (i a)=e .
$$

Comme $\exp (i a)=\sum_{j=1}^{r} e^{i a_{j}} c_{j}$, cette condition équivaut encore à $a_{j} \in 2 \pi \mathbb{Z}$ pour tout entier $j$ tel que $1 \leq j \leq r$. D'où le lemme.

Théorème 3.4. L'espace symétrique $S_{1}$ est simplement connexe.

Démonstration. Les lemmes 3.2 et 3.3 montrent que les réseaux $\Lambda_{0}$ et $\Lambda$ coïncident. Donc d'après [Lo, Theorem 3.6] (voir aussi $\left[\mathrm{He}_{2}, \mathrm{Ch}\right.$. VII. Theorem 8.4 et Theorem 9.1]), l'espace $S_{1}$ est bien simplement connexe.

Suivant une méthode classique, nous réalisons maintenant le revêtement universel de $S$. Soit

$$
\widetilde{S}=\left\{(\sigma, \theta) \in S \times \mathbb{R} \mid \operatorname{det}(\sigma)=e^{i r \theta}\right\},
$$

muni de la topologie induite par la topologie produit sur $S \times \mathbb{R}$. La projection sur le premier facteur réalise $\widetilde{S}$ comme un revêtement de $S$ avec fibre isomorphe à $\mathbb{Z}$.

Théorème 3.5. $\widetilde{S}$ est le revêtement universel de $S$.

Démonstration. Montrons en effet que l'application

$$
S_{1} \times \mathbb{R} \longrightarrow \widetilde{S} \quad(\sigma, \theta) \longmapsto\left(e^{i \theta} \sigma, \theta\right)
$$

est un homéomorphisme. D'abord l'application est bien à valeurs dans $\widetilde{S}$, car $\operatorname{det}\left(e^{i \theta} \sigma\right)=e^{i r \theta} \operatorname{det}(\sigma)=e^{i r \theta}$. Ensuite, l'application est clairement bijective, l'application réciproque étant $(\sigma, \theta) \longmapsto\left(e^{-i \theta} \sigma, \theta\right)$. De plus la continuité de l'application

\footnotetext{
${ }^{1}$ On devrait utiliser le produit scalaire induit par la forme de Killing, mais tout produit scalaire sur $\mathfrak{a}_{0}$ qui lui est proportionnel convient dans la détermination du réseau $\Lambda_{0}$.
} 
et de sa réciproque est immédiate. Comme $S_{1}$ est simplement connexe d'après le théorème 3.4 , l'espace $\widetilde{S}$ l'est aussi.

Nous allons maintenant construire un revêtement $\Gamma$ de $G$, puis une opération de $\Gamma$ sur $\widetilde{S}$ relevant l'action de $G$ sur $S$.

Soit $g \in G$ et $z \in \mathcal{D}$. On note $J(g, z)=D g(z)$ la différentielle de la transformation $z \longmapsto g(z)$. On sait que c'est un élément de $\mathbb{L}$, et on pose

$$
j(g, z)=\chi(J(g, z)) \in \mathbb{C}^{*} .
$$

Pour $g \in G$ fixé, on considère les déterminations de l'argument de $j(g, \cdot)$. Plus précisément une fonction $\varphi(g, \cdot): \mathcal{D} \longrightarrow \mathbb{R}$ est appelée une détermination de l'argument $j(g, \cdot)$ de si elle est continue et si

$$
\forall z \in \mathcal{D}, \quad e^{i \varphi(g, z)}=\frac{j(g, z)}{|j(g, z)|} .
$$

L'existence de telles déterminations est conséquence du fait que $\mathcal{D}$ est simplement connexe. Deux telles déterminations diffèrent d'un multiple entier de $2 \pi$.

On pose alors

$$
\Gamma=\left\{\left(g, \varphi_{g}\right) \mid g \in G\right\}
$$

où $g \in G$, et $\varphi_{g}=\varphi(g, \cdot)$ est une détermination de l'argument de $j(g, \cdot)$. La loi de groupe est donnée par

$$
(g, \varphi(g, \cdot))(h, \psi(h, \cdot))=(g h, \varphi(g, h(\cdot))+\psi(h, \cdot)) .
$$

Remarquons en effet que la formule

$$
J(g h, z)=J(g, h(z)) J(h, z)
$$

implique que

$$
e^{i[\varphi(g, h(z))+\psi(h, z)]}=e^{i \varphi(g, h(z))} e^{i \psi(h, z)}=\frac{j(g, h(z))}{|j(g, h(z))|} \frac{j(h, z)}{|j(h, z)|}=\frac{j(g h, z)}{|j(g h, z)|} .
$$

Par suite, $\varphi(g, h(\cdot))+\psi(h, \cdot)$ est bien une détermination de l'argument de $j(g h, \cdot)$.

Pour la topologie, on remarque qu'une détermination $\varphi(g, \cdot)$ de l'argument de $j(g, \cdot)$ est déterminée par sa valeur $\varphi(g, 0)$ à l'origine. Donc $\Gamma$ s'identifie ensemblistement au sous-ensemble fermé de $G \times \mathbb{R}$ donné par

$$
\left\{(g, \theta) \in G \times \mathbb{R} \mid e^{i \theta}=j(g, 0)\right\},
$$

et on munit $\Gamma$ de la topologie induite, faisant ainsi de $\Gamma$ un groupe topologique (la référence [God] contient les détails nécessaires dans le cas où $G=P S L(2, \mathbb{R}))$. La projection $\pi: \Gamma \longrightarrow G$ est continue, et fait de $\Gamma$ un revêtement de $G^{2}$.

L'application $\iota: \mathbb{Z} \longrightarrow \Gamma$ donnée par $\iota: n \longmapsto($ id, $2 n \pi)$ est un homomorphisme de groupes, et la suite exacte

$$
0 \longrightarrow \mathbb{Z} \stackrel{\iota}{\longrightarrow} \Gamma \stackrel{\pi}{\longrightarrow} G \longrightarrow 1
$$

est une extension centrale de $G$.

\footnotetext{
${ }^{2}$ On observera que le groupe $\Gamma$ n'est pas nécessairement connexe, comme on peut le voir dans le cas où $G=S p(E)$.
} 
Lemme 3.6. Soit $g \in G$, et $\sigma \in S$. Alors

$$
\operatorname{det}(g(\sigma))=\frac{j(g, \sigma)}{|j(g, \sigma)|} \operatorname{det}(\sigma) .
$$

Démonstration. Soit $G_{\sigma}$ le stabilisateur de $\sigma$ dans $G$. Comme $U$ est transitif sur $S$, on a $G=U G_{\sigma}$. L'élément $g$ peut donc s'écrire $g=u p$, avec $u \in U$ et $p \in G_{\sigma}$. Le membre de gauche de la formule (7) vaut

$$
\operatorname{det}(g(\sigma))=\operatorname{det}(u(\sigma))=\chi(u) \operatorname{det}(\sigma) .
$$

D'autre part, $j(g, \sigma)=j(u, \sigma) j(p, \sigma)$. Maintenant $J(p, \sigma)$ conserve globalement le plan tangent à $S$ en $\sigma$, qui est une forme réelle de $\mathbb{J}$, et conserve globalement le cône $C_{\sigma}$, et donc $j(p, \sigma)$ est un nombre réel strictement positif. D'autre par, l'action de $u$ étant linéaire, on a pour tout $z \in \mathbb{J}, J(u, z)=u$, et $j(u, z)=\chi(u)$. Par suite,

$$
\frac{j(g, \sigma)}{|j(g, \sigma)|}=\frac{j(u, \sigma)}{|j(u, \sigma)|} \frac{j(p, \sigma)}{|j(p, \sigma)|}=\chi(u)
$$

On peut maintenant définir une action de $\Gamma$ sur $\widetilde{S}$.

Proposition 3.7. Pour $(g, \varphi(g, \cdot)) \in \Gamma$ et $(\sigma, \theta) \in \widetilde{S}$, posons

$$
(g, \varphi(g, \cdot))(\sigma, \theta)=\left(g(\sigma), \theta+\frac{1}{r} \varphi(g, \sigma)\right) .
$$

On définit ainsi une action continue de $\Gamma$ sur $\widetilde{S}$.

Démonstration. On vérifie en effet que

$$
e^{i(r \theta+\varphi(g, \sigma))}=e^{i \varphi(g, \sigma)} e^{i r \theta}=\frac{j(g, \sigma)}{|j(g, \sigma)|} \operatorname{det}(\sigma)=\operatorname{det}(g(\sigma))
$$

d'après le lemme précédent, et donc $\left(g(\sigma), \theta+\frac{1}{r} \varphi(g, \sigma)\right)$ est bien élément de $\widetilde{S}$. Ensuite pour $(g, \varphi(g, \cdot))$ et $(h, \psi(h, \cdot)$ éléments de $\Gamma$, on a

$$
\begin{aligned}
(g, \varphi(g, \cdot)((h, \psi(h, \cdot))(\sigma, \theta)) & =(g, \varphi(g, \cdot))\left(h(\sigma), \theta+\frac{1}{r} \psi(g, \sigma)\right) \\
& =\left(g(h(\sigma)), \theta+\frac{1}{r} \psi(g, \sigma)+\frac{1}{r} \varphi(g, h(\sigma))\right. \\
& =(g h, \psi(g, \cdot)+\varphi(g, h \cdot))(\sigma, \theta) \\
& =((g, \varphi(g, \cdot)(h, \psi(h, \cdot))(\sigma, \theta)
\end{aligned}
$$

ce qui montre que l'on a bien à faire à une action (clairement continue) de $\Gamma$ sur $\widetilde{S}$.

Notons que l'élément $T=\iota(1)$ opère sur $\widetilde{S}$ par

$$
T \cdot(\sigma, \theta)=\left(\sigma, \theta+\frac{2 \pi}{r}\right) .
$$




\section{L'INDICE DE TRANSVERSALITÉ}

Nous allons introduire dans ce paragraphe un invariant qui caractérise les orbites de l'action de $G$ sur $S \times S$. Transporté par la transformation de Cayley, cet invariant est lié au rang des éléments de l'algèbre de Jordan $J$. Cette notion généralise la distance arithmétique étudiée par Hua $[\mathrm{Hu}]$ dans le cadre des algèbres de Jordan $\operatorname{Sym}(m, \mathbb{R})$ et $\operatorname{Herm}(m, \mathbb{C})$.

Rappelons d'abord la caractérisation des orbites de $L$ sur $J$. Soit $e=\sum_{j=1}^{r} c_{j}$ une décomposition de Peirce de l'identité. Pour tout couple d'entiers $0 \leq p, q \leq r$, tels que $0 \leq p+q \leq r$, on pose

$$
e_{p, q}=\sum_{j=1}^{p} c_{j}-\sum_{j=p+1}^{p+q} c_{j} .
$$

Notons $\mathcal{O}_{p, q}=L e_{p, q}$ l'orbite du point $e_{p, q}$ sous l'action du groupe $L$.

Théorème 4.1. L'espace $J$ est réunion disjointe des orbites $\mathcal{O}_{p, q}$, pour $0 \leq p, q \leq$ $r, p+q \leq r$.

Démonstration. (esquisse; pour une autre démonstration, voir [Ka]) Soit $x \in J$. Soient $\lambda_{j}, 1 \leq j \leq r$ ses valeurs propres, figurant autant de fois que leur multiplicité. Soit $p$ (resp. q) le nombre de valeurs propres strictement positives (resp. strictement négatives). D'après le théorème spectral, $x$ est conjugué par $K$ à l'élément $\sum_{j=1}^{p+q} \lambda_{j} c_{j}$, où on peut supposer de plus que $\lambda_{j}>0$ pour $1 \leq j \leq p$ et $\lambda_{j}<0$ pour $p+1 \leq j \leq p+q$. Grâce à l'action du sous-groupe $A=\exp L(\mathfrak{a})$, on voit que l'orbite de $x$ contient l'élément $e_{p, q}$. Donc $J$ est bien réunion des orbites $\mathcal{O}_{p, q}$.

Pour voir qu'elles sont disjointes, on observe que la signature de la forme quadratique $Q_{x}: y \longmapsto\langle P(x) y, y\rangle$ ne dépend que de l'orbite de $x$. Cela résulte de ce que $P(g x)=g P(x) g^{t}$ pour tout $g \in L$. Il suffit alors de vérifier que les signatures des formes quadratiques $Q_{p, q}$ données par $Q_{p, q}(y)=\left\langle P\left(e_{p, q}\right) y, y\right\rangle$ sont toutes distinctes.

En particulier, il y a $r+1$ orbites ouvertes, correspondant aux cas $p+q=r$, dont la réunion est l'ouvert $J^{\times}$des éléments inversibles de $J$.

Le rang d'un élément $x$ de $J$, noté $\operatorname{rang}(x)$, est par définition le nombre de ses valeurs propres non nulles comptées avec leur multiplicité (voir [F-K, Theorem III.1.1]). C'est un invariant sous l'action du groupe $L(\Omega)$. On observe que $x$ et $y$ ont même rang si et seulement si $P(x)$ et $P(y)$ ont même rang.

Soit $c$ un idempotent de $J$. La décomposition de Peirce de $J$ relativement à $c$ est (voir $[\mathrm{F}-\mathrm{K}$, chapter $\mathrm{IV}]$ )

$$
J=J(c, 1) \oplus J\left(c, \frac{1}{2}\right) \oplus J(c, 0) .
$$

Si $c$ est de rang $k$, alors

$$
\begin{aligned}
\operatorname{dim} J(c, 1) & =k+\frac{k(k-1)}{2} d, \\
\operatorname{dim} J(c, 0) & =(r-k)+\frac{(r-k)(r-k-1)}{2} d, \\
\operatorname{dim} J\left(c, \frac{1}{2}\right) & =k(r-k) d .
\end{aligned}
$$


Tout élément $x \in J$ se décompose relativement à (9) sous la forme

$$
x=x_{1}+x_{\frac{1}{2}}+x_{0} \text { avec } x_{0} \in J(c, 0), x_{1} \in J(c, 1), x_{\frac{1}{2}} \in J\left(c, \frac{1}{2}\right) .
$$

Les sous-espaces $J(c, 1)$ et $J(c, 0)$ sont des sous-algèbres de Jordan de $J$. On désigne par $\Omega_{1}$ (resp. $\left.\Omega_{0}\right)$ le cône symétrique associé à $J(c, 1)(\operatorname{resp} . J(c, 0))$ et on note $\operatorname{det}_{c}$ $\left(\right.$ resp. det $\left._{e-c}\right)$ le déterminant de $J(c, 1)(\operatorname{resp} . J(c, 0)=J(e-c, 1))$.

Voici quelques lemmes qui nous seront utiles dans la suite.

Lemme 4.2. Soit $x \in J$ un élément de rang $k$. Il existe un unique idempotent $c$ de rang $k$ tel que $c x=x$. De plus $c \in \mathbb{R}[x]$.

Démonstration. D'après le théorème spectral, (voir [F-K, Theorem III.1.1]), il existe un système complet d'idempotents orthogonaux ${ }^{3},\left(c_{j}\right)_{1 \leq j \leq m}$, un $m$-uplet de nombres réels $\left(\lambda_{j}\right)_{1 \leq j \leq m}$ deux à deux distincts tels que $x=\sum_{j=1}^{m} \lambda_{j} c_{j}$. Les $\left(c_{j}\right)_{1 \leq j \leq m}$ appartiennent à $\mathbb{R}[x]$, et les $\left(c_{j}, \lambda_{j}\right)_{1 \leq j \leq m}$ sont uniques à l'ordre près. Le rang de $x$ est la somme des rangs des idempotents $c_{j}$ pour lesquels $\lambda_{j} \neq 0$. L'élément

$$
c=\sum_{1 \leq j \leq m, \lambda_{j} \neq 0} c_{j}
$$

est un idempotent de même rang que $x$, et satisfait $c x=x$.

Réciproquement, soit $d$ un idempotent de $\operatorname{rang} k=\operatorname{rang}(x)$, tel que $d x=x$. Appliquant le théorème spectral dans l'algèbre $J(d, 1)$, on voit qu'il existe une famille unique d'idempotents $\left(d_{j}\right)_{1 \leq j \leq p}$ deux à deux orthogonaux, de somme $\sum_{j=1}^{p} d_{j}=d$ et des scalaires uniques $\left(\mu_{j}\right)_{1 \leq j \leq p}$ deux à deux distincts tels que $x=\sum_{j=1}^{d} \mu_{j} d_{j}$. De plus aucun des $\mu_{j}$ n'est nul, puisque $\operatorname{rang}(x)=\operatorname{rang}(d)$ par hypothèse. Soit $d_{p+1}=e-d$. Alors $\sum_{j=1}^{p+1} d_{j}=e$ et on peut écrire

$$
x=\sum_{j=1}^{p} \mu_{j} d_{j}+0 d_{p+1} .
$$

D'après l'assertion d'unicité dans le théorème spectral, on voit que $m=p+1$, et qu'à l'ordre près, les $d_{j}\left(\right.$ resp. $\left.\mu_{j}\right)$ coïncident avec les $c_{j}\left(\right.$ resp. $\left.\lambda_{j}\right)$. D'où facilement $c=d$.

Lemme 4.3. Soit $x=x_{1}+x_{\frac{1}{2}}+x_{0}$ la décomposition de Peirce d'un élément $x \in J$ relativement ̀̀ un idempotent $c \in J$. Si $x \in \Omega$, alors $x_{1} \in \Omega_{1}$ et $x_{0} \in \Omega_{0}$.

Démonstration. Rappelons d'abord une caractérisation du cône $\Omega$ (voir $[\mathrm{F}-\mathrm{K}$, page $4]$ ). Pour tout $x \in J$, on a l'équivalence suivante

$$
x \in \Omega \Longleftrightarrow \forall y \in \bar{\Omega} \backslash\{0\},\langle x \mid y\rangle>0 \text {. }
$$

On a une caractérisation analogue pour $\Omega_{1} \subset J(c, 1)$, que nous allons utiliser pour démontrer le lemme. Soit donc $y_{1} \in \bar{\Omega}_{1} \backslash\{0\}$. D'après le théorème spectral relativement à $J(c, 1)$ (voir $[\mathrm{F}-\mathrm{K}$, Theorem III.1.2]), il existe une décomposition de Peirce de l'unité $c=\sum_{j=1}^{k} c_{j}$ telle que l'on puisse écrire

$$
y_{1}=\sum_{j=1}^{k} \lambda_{j} c_{j},
$$

\footnotetext{
${ }^{3}$ non nécessairement primitifs
} 
les $\lambda_{j}, 1 \leq j \leq k$ étant positifs ou nuls et non tous nuls. Il en résulte en particulier que $y_{1} \in \bar{\Omega} \backslash\{0\}$. En utilisant l'orthogonalité de la décomposition (9) on voit que

$$
\left\langle x_{1} \mid y_{1}\right\rangle=\left\langle x_{1}+x_{\frac{1}{2}}+x_{0} \mid y_{1}\right\rangle=\left\langle x \mid y_{1}\right\rangle>0
$$

Ceci étant vrai pour tout $y_{1} \in \bar{\Omega}_{1} \backslash\{0\}$, on a bien $x \in \Omega_{1}$. Pour montrer que $x_{0} \in \Omega_{0}$, il suffit de considérer la décomposition de Peirce par rapport à l'idempotent $e-c$.

Lemme 4.4. Soit c un idempotent de J. Pour tout $x_{1} \in J(c, 1)$ et tout $x_{0} \in J(c, 0)$ on $a$

$$
\operatorname{det}\left(x_{1}+x_{0}\right)=\operatorname{det}_{c}\left(x_{1}\right) \operatorname{det}_{e-c}\left(x_{0}\right) .
$$

Démonstration. Soit $k$ le rang de $c$. Alors $J(c, 1)$ et $J(c, 0)=J(e-c, 1)$ sont deux algèbres de Jordan de rang $k$ et $r-k$ respectivement. Il existe alors $\left\{c_{1}, \ldots, c_{k}\right\}$ et $\left\{c_{k+1}, \ldots, c_{r}\right\}$ deux repères de Jordan de $J(c, 1)$ et $J(c, 0)$ respectivement tels que $x_{1}=\sum_{j=1}^{k} \lambda_{j} c_{j}$ et $x_{0}=\sum_{j=k+1}^{r} \lambda_{j} c_{j}$ soient les décompositions spectrales de $x_{1} \in J(c, 1)$ et $x_{0} \in J(c, 0)$. Or $\left\{c_{1} \ldots, c_{k}, c_{k+1}, \ldots, c_{r}\right\}$ est un repère de Jordan de $J$, donc $\sum_{j=1}^{r} \lambda_{j} c_{j}$ est la décomposition spectrale de $x_{1}+x_{0}$ dans $J$. On en déduit que

$$
\operatorname{det}\left(x_{1}+x_{0}\right)=\left(\prod_{j=1}^{k} \lambda_{j}\right)\left(\prod_{j=k+1}^{r} \lambda_{j}\right)=\operatorname{det}_{c}\left(x_{1}\right) \operatorname{det}_{e-c}\left(x_{0}\right) .
$$

Pour tout $z, w \in J$, on introduit l'opérateur

$$
z \square w=L(z w)+[L(z), L(w)] .
$$

À chaque $z$ de $J\left(c, \frac{1}{2}\right)$ est associé la transformation de Frobenius

$$
\tau_{c}(z)=\exp (2 z \square c)
$$

qui est un élément du groupe $L$.

Si $x=x_{1}+x_{\frac{1}{2}}+x_{0}$ est la décomposition de Peirce de $x \in J$ par rapport à $c$, alors (voir [F-K, Lemma VI.3.1]) $\tau_{c}(z) x=y_{1}+y_{\frac{1}{2}}+y_{0}$, où

$$
\begin{aligned}
y_{1} & =x_{1} \\
y_{\frac{1}{2}} & =2 L(z) x_{1}+x_{\frac{1}{2}} \\
y_{0} & =2 L(e-c)\left[L(z)^{2} x_{1}+L(z) x_{\frac{1}{2}}\right]+x_{0}
\end{aligned}
$$

On en déduit que $\operatorname{Det}\left(\tau_{c}(z)\right)=1$. Soit $\tau_{c}(z)^{*}$ l'adjoint de la transformation de Frobenius $\tau_{c}(z)$ par rapport au produit scalaire (1). On a

$$
\tau_{c}(z)^{*}=\exp (2 c \square z)=\exp (2 z \square(e-c))=\tau_{e-c}(z) .
$$

Si $u=u_{1}+u_{\frac{1}{2}}+u_{0}$ est la décomposition de Peirce d'un élément $u \in J$ par rapport à $c$, alors la décomposition $\tau_{c}(z)^{*} u=v_{1}+v_{\frac{1}{2}}+v_{0}$ est donnée par

$$
\begin{aligned}
v_{1} & =2 L(c)\left[L(z)^{2} u_{0}+L(z) u_{\frac{1}{2}}\right]+u_{1} \\
v_{\frac{1}{2}} & =2 L(z) u_{0}+u_{\frac{1}{2}} \\
v_{0} & =u_{0}
\end{aligned}
$$


En particulier, la restriction de $\tau_{c}(z)^{*}$ à $J(c, 1)$ coïncide avec l'identité.

Montrons maintenant le lemme suivant qui généralise la proposition VI.3.2 dans [F-K, page 107] à un idempotent $c$ quelconque.

Lemme 4.5. Soit c un idempotent quelconque de $J$, et soit $J=J(c, 1) \oplus J\left(c, \frac{1}{2}\right) \oplus$ $J(c, 0)$ la décomposition de $J$ associée. Soit $\omega \in \Omega$ et soit $\omega=\omega_{1}+\omega_{\frac{1}{2}}+\omega_{0}$ sa décomposition correspondante. Alors il existe un unique $z \in J\left(c, \frac{1}{2}\right)$ tel que

$$
\omega=\tau_{c}(z)\left(\omega_{1}+\xi_{0}\right)
$$

où $\xi_{0}$ est un élément du cône symétrique $\Omega_{0}$ associé à l'algèbre de Jordan $J(c, 0)$.

Démonstration. L'algèbre de Jordan $J(c, 1)$ agit par multiplication sur $J\left(c, \frac{1}{2}\right)$, et l'application $\Phi$ de $J(c, 1)$ dans $\operatorname{End}\left(J\left(c, \frac{1}{2}\right)\right)$ définie par

$$
\forall x \in J(c, 1), \forall \xi \in J\left(c, \frac{1}{2}\right), \Phi(x) \xi=2 x \xi
$$

est une représentation de l'algèbre de Jordan $J(c, 1)$ sur $J\left(c, \frac{1}{2}\right)$.

Si $z \in J\left(c, \frac{1}{2}\right)$ alors d'après les relations (11)

$$
\begin{aligned}
\tau_{c}(-z) \omega & =\omega_{1}-2 L(z) \omega_{1}+\omega_{\frac{1}{2}}+\xi_{0} \\
& =\omega_{1}-\Phi\left(\omega_{1}\right) z+\omega_{\frac{1}{2}}+\xi_{0}
\end{aligned}
$$

où $\xi_{0} \in J(c, 0)$. Du fait que $\omega \in \Omega$, d'après le lemme 4.3 , la composante $\omega_{1}$ est inversible dans $J(c, 1)$ et par suite l'endomorphisme $\Phi\left(\omega_{1}\right)$ est inversible dans $\operatorname{End}\left(J\left(c, \frac{1}{2}\right)\right)$. La proposition s'en déduit en choisissant $z=\Phi\left(\omega_{1}\right)^{-1} \omega_{\frac{1}{2}}$.

Lemme 4.6. Soit $x \in J$ un élément de rang $k, c$ l'unique idempotent de rang $k$ tel que $c x=x$, et soit $J=J(c, 1) \oplus J\left(c, \frac{1}{2}\right) \oplus J(c, 0)$ la décomposition de $J$ associée à l'idempotent $c$. Soit $\omega \in \Omega$ et soit $\omega=\omega_{1}+\omega_{\frac{1}{2}}+\omega_{0}$ sa décomposition correspondante. Alors

$$
\operatorname{det}(x+t \omega)=t^{r-k} \operatorname{det}_{c}(x) \operatorname{det}_{e-c}\left(\omega_{0}\right)+O\left(t^{r-k+1}\right) .
$$

Démonstration. D'après le lemme 4.5 il existe $z \in J\left(c, \frac{1}{2}\right)$ tel que $\omega=\tau_{c}(z)^{*}\left(\omega_{0}+\right.$ $\left.\xi_{1}\right)$, avec $\xi_{1} \in \Omega_{1}$. Comme on a de plus $\tau_{c}(z)^{*} x=x$, il vient

$$
x+t \omega=\tau_{c}(z)^{*}\left(\left(x+t \xi_{1}\right)+t \omega_{0}\right)
$$

et donc d'après le lemme 4.4

$$
\operatorname{det}(x+t \omega)=\operatorname{det}_{c}\left(x+t \xi_{1}\right) t^{r-k} \operatorname{det}_{e-c}\left(\omega_{0}\right)
$$

d'où le résultat.

Corollaire 4.7. Soit $x \in J$ un élément de rang $k$ et soit $\Phi_{x}$ la fonction définie sur $J \operatorname{par} \Phi_{x}(z)=\operatorname{det}(x+z)$. Alors,

(i) pour tout $j, 1 \leq j \leq r-k-1, D^{(j)} \Phi_{x}(0)=0$.

(ii) la fonction $\frac{1}{(r-k) !} D^{(r-k)} \Phi_{x}(0) z=\Psi_{0}(z)$ est un polynôme homogène de degré $r-k$ qui ne s'annule pas sur $\Omega$. 
Lemme 4.8. Soit $x \in J$, et soit $K$ un compact contenu dans $\Omega$. Il existe une constante $\epsilon>0$ (dépendant de $x$ et de $K$ ) telle que pour tout $t, 0<t \leq \epsilon$ et tout $\omega \in K$ on ait

$$
\operatorname{det}(x+t \omega) \neq 0 .
$$

Démonstration. D'après le lemme 4.6, on peut écrire

$$
\operatorname{det}(x+t \omega)=t^{r-k} \Psi_{0}(\omega)+\sum_{j=1}^{k} t^{r-k+j} \Psi_{j}(\omega)
$$

où les $\Psi_{j}$ sont des polynômes homogènes de degré $r-k+j$. La fonction $\Psi_{0}$ garde

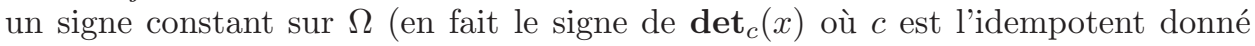
par le lemme 4.2). On va supposer qu'on est dans le cas où ce signe est + , l'autre cas se traitant de la même manière. Soit $c_{0}>0$ le minimum de $\Psi_{0}$ sur le compact $K$, et soit $C$ une constante positive telle que pour tout $j, 1 \leq j \leq k$ on ait

$$
\forall \omega \in K, \quad\left|\Psi_{j}(\omega)\right| \leq C .
$$

On a alors pour $\omega \in K$ et $t>0$

$$
\operatorname{det}(x+t \omega)>t^{r-k}\left(c_{0}-C \sum_{j=1}^{k} t^{j}\right) .
$$

Posons $\epsilon=\inf \left(1, \frac{1}{2} \frac{c_{0}}{k C}\right)$. Alors pour $0 \leq t \leq \epsilon$, on a

$$
c_{0}-C \sum_{j=1}^{k} t^{j} \geq c_{0}-C k \epsilon \geq \frac{1}{2} c_{0}>0 .
$$

D'où pour $\omega \in K$ et $0<t \leq \epsilon, \operatorname{det}(x+t \omega)>0$.

Proposition 4.9. Soit $\gamma(t), 0 \leq t \leq 1$ une courbe causale d'origine $x \in J$.

(i) Il existe $\epsilon>0$ tel que $\forall t, 0<t \leq \epsilon$, $\operatorname{det}(\gamma(t)) \neq 0$.

(ii) Si $x$ est de rang $k$, il existe une constante $a_{k} \neq 0$ telle que

$$
\operatorname{det}(\gamma(t))=a_{k} t^{r-k}+O\left(t^{r-k+1}\right) .
$$

Démonstration. Soit $C=\{\dot{\gamma}(t), 0 \leq t \leq 1\}$. Alors $C$ est un compact de $\Omega$, et son enveloppe convexe $\Gamma(C)$ également. Écrivant

$$
\gamma(t)=t \int_{0}^{1} \dot{\gamma}(s t) d s
$$

on voit que $\gamma(t)=t \omega(t)$, où $\omega(t) \in \Gamma(C)$. La partie $(i)$ est donc conséquence du lemme 4.8.

La partie (ii) est conséquence du corollaire 4.7, (ii).

Definition 4.10. Soient $x$ et $y$ deux éléments quelconques de J. La quantité

$$
\mu(x, y)=r-\operatorname{rang}(x-y)
$$

est appelé l'indice de transversalité de la paire $(x, y)$. 
Les deux cas extrêmes sont le cas où $x-y$ est inversible, auquel cas l'indice de transversalité vaut 0 , et le cas où $x=y$, auquel cas l'indice de transversalité vaut $r$.

On va maintenant montrer que l'indice de transversalité caractérise les orbites de $G\left(T_{\Omega}\right)$ dans $J \times J$.

Remarque 4.11. D'après (6), le groupe conforme $G\left(T_{\Omega}\right)$ agit de manière rationnelle sur l'algèbre de Jordan $J$ si on la réalise dans sa compactification conforme $G\left(T_{\Omega}\right) / L N^{-}$. On peut alors montrer que $\left\{g \in G\left(T_{\Omega}\right) \mid g(0) \in J\right\}=N^{+} L N^{-}$et que le stabilisateur de 0 dans $G\left(T_{\Omega}\right)$ est $L N^{-}$. Pour toutes ses considérations voir [Ko].

Théorème 4.12. L'indice de transversalité sur $J$ est invariant sous l'action $d u$ groupe conforme $G\left(T_{\Omega}\right)$. Plus précisément, si g est une transformation conforme de $J$ qui est définie en $x$ et $y$, alors

$$
\mu(g(x), g(y))=\mu(x, y) .
$$

Démonstration. Il est immédiat que l'indice de transversalité est invariant par translation. Comme le groupe $L$ conserve le rang d'un élément (conséquence du théorème 4.1), il conserve l'indice de transversalité. Reste donc à voir l'invariance sous l'action de l'inversion. Soit donc $x, y$ deux éléments inversibles de $J$. On utilise alors l'identité de Hua sous la forme

$$
P\left(x^{-1}-y^{-1}\right)=P(x)^{-1} P(x-y) P(y)^{-1} .
$$

On en déduit que $P\left(x^{-1}-y^{-1}\right)$ et $P(x-y)$ ont même rang, mais cela est équivalent à dire (voir [F-K, Proposition IV.3.1]) que $x^{-1}-y^{-1}$ et $x-y$ ont même rang. D'où le résultat.

Abordons maintenant la réciproque.

Lemme 4.13. Soient $x, x^{\prime} \in J$ tels que $\mu(x, 0)=\mu\left(x^{\prime}, 0\right)$. Alors il existe $h \in L N^{-}$ tel que $h(x)=x^{\prime}$.

Démonstration. Posons $\mu(x, 0)=\mu\left(x^{\prime}, 0\right)=k$. L'élément $x$ est donc de rang $\ell=$ $r-k$. Il existe un repère de Jordan $\left(c_{j}\right)_{1 \leq j \leq r}$ et des nombres réels non nuls $\left(\lambda_{j}\right)_{1 \leq j \leq \ell}$ tels que $x=\sum_{j=1}^{\ell} \lambda_{j} c_{j}$. Pour $\epsilon>0$ assez petit, l'élément

$$
x_{\epsilon}=\sum_{j=1}^{\ell} \lambda_{j} c_{j}+\sum_{j=\ell+1}^{r} \epsilon c_{j}
$$

est inversible. Son image par $\widetilde{t}_{v}$ où $v=\sum_{j=1}^{\ell} \frac{\lambda_{j}-1}{\lambda_{j}} c_{j}$ est donnée par

$$
\begin{aligned}
\widetilde{t}_{v}\left(x_{\epsilon}\right) & =\left(s \circ t_{v} \circ s\right)\left(x_{\epsilon}\right) \\
& =\left(x_{\epsilon}^{-1}+v\right)^{-1} \\
& =\sum_{j=1}^{\ell} c_{j}+\sum_{j=\ell+1}^{r} \epsilon c_{j} .
\end{aligned}
$$

(voir (4) et (5) pour ces différentes notations). Comme $\widetilde{t}_{v}$ est continue au voisinage de tout élément inversible, $\widetilde{t}_{v}\left(x_{\varepsilon}\right)$ tends vers $\sum_{j=1}^{\ell} c_{j}$ lorsque $\varepsilon$ tend vers 0 . Par 
prolongement par continuité on a donc

$$
\widetilde{t}_{v}(x)=\sum_{j=1}^{\ell} c_{j} .
$$

L'élément $x^{\prime}$ est aussi de rang $\ell$, et il existe un système de Jordan $\left(c_{j}^{\prime}\right)_{1 \leq r}$ et des nombres réels non nuls $\left(\lambda_{j}^{\prime}\right)_{1 \leq j \leq \ell}$ tels que $x^{\prime}=\sum_{j=1}^{\ell} \lambda_{j}^{\prime} c_{j}^{\prime}$. On montre de même qu'il existe une transformation $\widetilde{t}_{v^{\prime}}$ telle que

$$
\widetilde{t}_{v^{\prime}}\left(x^{\prime}\right)=\sum_{j=1}^{\ell} c_{j}^{\prime} .
$$

D'après [F-K, Theorem IV.2.5], il existe un automorphisme $k \in A u t(J)$ tel que $k\left(c_{j}\right)=c_{j}^{\prime}$ pour tout $j, 1 \leq j \leq r$. L'élément $h=\widetilde{t}_{v^{\prime}}^{-1} \circ k \circ \widetilde{t}_{v} \in L N^{-}$vérifie alors $h(x)=x^{\prime}$.

Théorème 4.14. Soient $x, x_{0}, y, y_{0} \in J$. Si $\mu\left(x, x_{0}\right)=\mu\left(y, y_{0}\right)$, alors il existe un élément $g \in N^{+} L N^{-}$tel que $y=g(x)$ et $y_{0}=g\left(x_{0}\right)$.

Démonstration. La condition $\mu\left(x, x_{0}\right)=\mu\left(y, y_{0}\right)$ étant équivalente à $\mu\left(x-x_{0}, 0\right)=$ $\mu\left(y-y_{0}, 0\right)$, les deux éléments $x-x_{0}$ et $y-y_{0}$ ont le même rang. D'après le lemme 4.13 , il existe $h \in L N^{-}$tel que $y-y_{0}=h\left(x-x_{0}\right)$. L'élément $g=t_{y_{0}} \circ h \circ t_{-x_{0}} \in$ $N^{+} L N^{-}$vérifie $g(x)=y$ et $g\left(x_{0}\right)=y_{0}$ puisque $h$ fixe 0 d'après la remarque 4.11.

Rappelons la définition de la transversalité introduite dans $\left[\mathrm{C}-\varnothing_{1}\right]$. On dira que deux éléments $\sigma$ et $\tau$ de $S$ sont transverses si,

$$
\sigma \top \tau \stackrel{\text { def }}{\Longleftrightarrow} \operatorname{det}(\sigma-\tau) \neq 0 .
$$

Pour étendre la définition de l'indice de transversalité à $S \times S$ nous avons besoin de la proposition suivante.

Proposition 4.15. Soient $\sigma, \tau \in S$, alors il existe un élément $u$ de $U$ tel que $u(\sigma)$ et $u(\tau)$ soient transverses à e. De plus l'entier

$$
\mu(c(u(\sigma)), c(u(\tau)))
$$

ne dépend pas de l'élément $u \in U$ choisi.

Démonstration. Soient $\sigma, \tau \in S$ et soit $\varsigma \in S$ tel que $\varsigma \top \sigma$ et $\varsigma \top \tau$. On peut trouver $u \in U$, vérifiant $u(\varsigma)=e$. Les deux éléments $u(\sigma)$ et $u(\tau)$ sont alors transverses à $e$ et par conséquent appartiennent à $p(J)$.

Supposons maintenant qu'il existe deux éléments $u_{1}, u_{2} \in U$ tels que $u_{i}(\sigma)$ et $u_{i}(\tau)$ soient transverses à $e$ pour $i=1,2$. Posons $c\left(u_{i}(\sigma)\right)=x_{i}$ et $c\left(u_{i}(\tau)\right)=y_{i}$, pour $i=1,2$. Alors $x_{2}=\left(c u_{2} u_{1}^{-1} c^{-1}\right)\left(x_{1}\right)$ et $y_{2}=\left(c u_{2} u_{1}^{-1} c^{-1}\right)\left(y_{1}\right)$. Comme $c u_{2} u_{1}^{-1} c^{-1} \in$ $G\left(T_{\Omega}\right)$, on a d'après (14),

$$
\begin{aligned}
\mu\left(x_{1}, y_{1}\right) & =\mu\left(\left(c u_{2} u_{1}^{-1} c^{-1}\right)\left(x_{1}\right),\left(c u_{2} u_{1}^{-1} c^{-1}\right)\left(y_{1}\right)\right), \\
& =\mu\left(x_{2}, y_{2}\right) .
\end{aligned}
$$

Ceci justifie la définition suivante 
Definition 4.16. Soit $(\sigma, \tau) \in S \times S$. On appelle indice de transversalité de la paire $(\sigma, \tau)$ l'indice de transversalité de la paire $(c(u(\sigma)), c(u(\tau))) \in J \times J$ où $u \in U$ est choisi tel que $u(\sigma) \top$ e et $u(\tau) \top e$. On note

$$
\mu(\sigma, \tau)=\mu(c(u(\sigma)), c(u(\tau))) .
$$

En particulier, $\sigma$ et $\tau$ sont transverses si et seulement si $\mu(\sigma, \tau)=0$.

Remarque 4.17. On peut montrer que

$$
\mu(\sigma, \tau)=k \Longleftrightarrow \operatorname{corang} P(\sigma-\tau)=k+\frac{k(k-1)}{2} d .
$$

Proposition 4.18. L'indice de transversalité sur $S$ est invariant par le groupe $G$,

$$
\mu(g(\sigma), g(\tau))=\mu(\sigma, \tau), \quad \forall g \in G .
$$

Démonstration. Cela résulte du théorème 4.12 et du fait que $G\left(T_{\Omega}\right)=c \circ G \circ c^{-1}$.

Théorème 4.19. L'indice de transversalité caractérise les orbites de l'action de $G$ sur $S \times S$. Plus précisément, si $\left(\sigma, \sigma_{0}\right),\left(\tau, \tau_{0}\right)$ sont deux éléments de $S \times S$ et si $\mu\left(\sigma, \sigma_{0}\right)=\mu\left(\tau, \tau_{0}\right)$ alors il existe $g \in G$ tel que $g(\sigma)=\tau$ et $g\left(\sigma_{0}\right)=\tau_{0}$.

Démonstration. Remarquons d'abord que d'après l'invariance de $\mu$ on peut supposer $\sigma_{0}=\tau_{0}=e$. Il suffit alors de montrer que si $\mu(\sigma, e)=\mu(\tau, e)$ alors il existe $g \in G$ tel que $g(\sigma)=\tau$ et $g(e)=e$. Soit $\varsigma \in S$ tel que $\varsigma \top \sigma, \varsigma \top \tau$ et $\varsigma \top e$. Soit $u \in U$ tel que $u(\sigma) \in p(J), u(\tau) \in p(J)$ et $u^{-1}(e)=\varsigma$. Posons $x=c(u(\sigma)), y=c(u(\tau))$ et $z=c(u(e))$. La condition $\mu(\sigma, e)=\mu(\tau, e)$ se traduit par $\mu(x, z)=\mu(y, z)$. La proposition 4.14 montre qu'il existe $h \in N^{+} L N^{-}$tel que $y=h(x)$ et $z=h(z)$. Donc l'élément $g=u^{-1} c^{-1} h c u \in G$ vérifie $g(\sigma)=\tau$ et $g(e)=e$. D'où le théorème.

Proposition 4.20. Soit $\sigma, \tau \in S$ et $[0,1] \ni t \mapsto \tau(t) \in S$ une courbe causale d'origine $\tau$. Il existe $\epsilon>0$ tel que $\forall t, 0<t \leq \epsilon, \tau(t)$ est transverse à $\sigma$.

Démonstration. Le résultat se déduit par transformation de Cayley de la proposition 4.9 .

\section{L'INDice DE Souriau : CAS TRANSVERSE}

Des résultats précédents, on déduit que le groupe $G$ préserve la transversalité, et agit transitivement sur

$$
S_{\top}^{2}=\left\{(\sigma, \tau) \in S^{2} \mid \sigma \top \tau\right\} .
$$

Soit $S_{\top}(-e)$ l'ouvert défini par

$$
S_{\top}(-e)=\{\sigma \in S \mid \sigma \top(-e)\} .
$$

Si $\sigma \in S_{\top}(-e)$, alors $(\sigma-s e)$ est inversible pour $s=-1$, et donc pour tout $s \in(-\infty, 0]$. L'intégrale (à valeurs dans $\mathbb{J}$ )

$$
\log \sigma=\int_{-\infty}^{0}\left((s e-\sigma)^{-1}-(s-1)^{-1} e\right) d s
$$

est normalement convergente, et définit une fonction régulière sur l'ouvert $S_{\top}(-e)$.

Proposition 5.1. Pour tout $\sigma \in S_{\top}(-e)$, on a

(i) $\exp (\log \sigma)=\sigma$. 
(ii) $e^{\operatorname{tr}(\log \sigma)}=\operatorname{det}(\sigma)$.

(iii) $\log \sigma^{-1}=-\log \sigma$.

(iv) $\log (k \sigma)=k \log \sigma$, pour tout $k \in \operatorname{Aut}(J)$.

Démonstration. Soit $\sigma \in S_{\top}(-e)$. Il existe une décomposition de Peirce $e=\sum_{j=1}^{r} c_{j}$ de l'identité adaptée à $\sigma$, c'est-à-dire telle que

$$
\sigma=\sum_{j=1}^{r} \sigma_{j} c_{j}, \quad\left|\sigma_{j}\right|=1,1 \leq j \leq r .
$$

Par hypothèse, $\sigma_{j} \neq-1$ pour tout $j$ tel que $1 \leq j \leq r$, et pour $s \in(-\infty, 0]$

$$
(s e-\sigma)^{-1}=\sum_{j=1}^{r}\left(s-\sigma_{j}\right)^{-1} c_{j} .
$$

Par suite,

$$
\log \sigma=\sum_{j=1}^{r}\left(\int_{-\infty}^{0}\left(\left(s-\sigma_{j}\right)^{-1}-(s-1)^{-1}\right) d s\right) c_{j} .
$$

On est donc ramené au calcul, pour $z \in \mathbb{C} \backslash(-\infty, 0]$ de l'intégrale

$$
\int_{-\infty}^{0}\left((s-z)^{-1}-(s-1)^{-1}\right) d s .
$$

Pour $z$ réel strictement positif, un calcul élémentaire montre que cette intégrale vaut $\log z$. Par prolongement analytique, on obtient que l'intégrale coïncide avec la détermination principale de $\log z$, pour tout $z \in \mathbb{C} \backslash(-\infty, 0]$. Donc, avec la même convention

$$
\log \sigma=\sum_{j=1}^{r} \log \sigma_{j} c_{j}
$$

d'où $(i)$ en calculant l'exponentielle des deux membres. Les autres points de la proposition se déduisent de (15).

Soit maintenant $\sigma$ et $\tau$ deux éléments transverses de $S$. Il existe un élément $u \in U$ tel que $\tau=u(-e)$. L'élément $u$ n'est pas unique, mais tout autre élément $u^{\prime}$ satisfaisant $\tau=u^{\prime}(-e)$ peut s'écrire $u^{\prime}=u k$ où $k \in U_{-e}=U_{e}=U \cap \operatorname{Aut}(J)$. L'élément $u^{-1}(\sigma)$ est transverse à $-e$, et donc on peut définir $\log u^{-1}(\sigma)$. Pour $u^{\prime}=$ $u k$, on obtient $\log u^{\prime-1}(\sigma)=k^{-1} \log u^{-1}(\sigma)$ et donc $\operatorname{tr}\left(\log u^{-1}(\sigma)\right)$ est indépendant du représentant $u$ choisi. En conséquence, on pose

$$
\Psi(\sigma, \tau)=\frac{1}{i} \operatorname{tr}\left(\log u^{-1}(\sigma)\right) .
$$

On définit ainsi une fonction sur $S_{\text {T }}^{2}$ une fonction à valeurs réelles. De plus,

$$
e^{\operatorname{tr}\left(\log u^{-1}(\sigma)\right)}=\operatorname{det}\left(u^{-1}(\sigma)\right)=\chi(u)^{-1} \operatorname{det}(\sigma)=(-1)^{r}(\operatorname{det} \sigma)(\operatorname{det} \tau)^{-1}
$$

de sorte que

$$
e^{2 i \Psi(\sigma, \tau)}=(\operatorname{det} \sigma)^{2}(\operatorname{det} \tau)^{-2} .
$$

Proposition 5.2. La fonction $\Psi: S_{\top}^{2} \rightarrow \mathbb{R}$ est régulière. Soient $\sigma, \tau \in S_{\top}^{2}$. Alors

(i) $\Psi(v(\sigma), v(\tau))=\Psi(\sigma, \tau)$, pour tout $v \in U$. 
(ii) $\Psi(\sigma, \tau)=-\Psi(\tau, \sigma)$.

Démonstration. Montrons la continuité de $\Psi$ en un point $\left(\sigma_{0}, \tau_{0}\right) \in S_{\text {T }}^{2}$. Il est clair que l'élément $u \in U$ tel que $\tau=u(-e)$ peut être choisi continûment pour $\tau$ proche de $\tau_{0}$. Il suffit en effet d'utiliser le fait que $S$ est un espace symétrique sous l'action de $U$, et donc il y a une section locale régulière de l'application $U \rightarrow S, v \mapsto v^{-1}\left(\tau_{0}\right)$ qui vaut $u$ en $-e$. D'après le théorème de continuité de l'intégrale d'une fonction dépendant de paramètres, il s'en suit que $\Psi(\sigma, \tau)$ est bien une fonction continue de $(\sigma, \tau)$ au voisinage de $\left(\sigma_{0}, \tau_{0}\right)$. La différentiabilité de $\Psi$ se démontre de même.

Soit $v \in U$. Alors, si $\tau=u(-e)$, on a $v(\tau)=(v \circ u)(-e)$, de sorte que

$$
\Psi(v(\sigma), v(\tau))=\frac{1}{i} \operatorname{tr}\left[\log \left((v \circ u)^{-1}(v(\sigma))\right)\right]=\frac{1}{i} \operatorname{tr}\left(\log u^{-1}(\sigma)\right)=\Psi(\sigma, \tau) .
$$

Pour démontrer $(i i)$, on peut compte-tenu de $(i)$ supposer que $\tau=-e$. Choisissons une racine carrée $\sigma^{\frac{1}{2}}$ de $\sigma$. Comme $i \sigma^{\frac{1}{2}} \in S$, l'élément $P\left(i \sigma^{\frac{1}{2}}\right)$ appartient à $U$, et satisfait $P\left(i \sigma^{\frac{1}{2}}\right)(-e)=\sigma$. Par suite

$\Psi(-e, \sigma)=\frac{1}{i} \operatorname{tr}\left[\log \left(P\left(i \sigma^{\frac{1}{2}}\right)^{-1}(-e)\right)\right]=\frac{1}{i} \operatorname{tr}\left(\log \sigma^{-1}\right)=-\frac{1}{i} \operatorname{tr}(\log \sigma)=-\Psi(\sigma,-e)$

où on a utilisé le fait que $\sigma^{-1}=\bar{\sigma}$ pour tout élément de $S$. D'où (ii).

Pour $\theta \in \mathbb{R}, \theta \notin \pi+2 \pi \mathbb{Z}$, on note $\{\theta\}$ le réel caractérisé par

$$
\{\theta\} \equiv \theta[2 \pi], \quad-\pi<\{\theta\}<\pi
$$

(une version de la détermination principale de $\theta$ ).

Proposition 5.3. Soit $\left(c_{j}\right)_{1 \leq j \leq r}$ une repère de Jordan de $J$, et soit

$$
\sigma=\sum_{j=1}^{r} e^{i \theta_{j}} c_{j}, \quad \tau=\sum_{j=1}^{r} e^{i \phi_{j}} c_{j}
$$

deux éléments transverses de $S$ (c'est-à-dire tels que $\theta_{j} \not \equiv \phi_{j}[2 \pi]$ pour tout $j$, $1 \leq j \leq r)$. Alors

$$
\Psi(\sigma, \tau)=\sum_{j=1}^{r}\left\{\theta_{j}-\phi_{j}+\pi\right\}
$$

Démonstration. Posons $u=\exp \left[i L\left(\sum_{j=1}^{r}\left(\phi_{j}-\pi\right) c_{j}\right)\right] \in U$. On a $u(-e)=\tau$ et $u^{-1}(\sigma)=\sum_{j=1}^{r} e^{i\left(\theta_{j}-\phi_{j}+\pi\right)} c_{j}$. D'où le résultat, d'après (15).

On étend la relation de transversalité à $\widetilde{S}$, en posant, pour deux éléments $(\sigma, \theta),(\tau, \phi)$ de $\widetilde{S}$

$$
(\sigma, \theta) \top(\tau, \phi) \Longleftrightarrow \sigma \top \tau
$$

et on note $\widetilde{S}_{\top}^{2}=\left\{(\widetilde{\sigma}, \widetilde{\tau}) \in \widetilde{S}^{2} \mid \widetilde{\sigma} \top \widetilde{\tau}\right\}$. Pour un couple d'éléments transverses $\widetilde{\sigma}=(\sigma, \theta), \widetilde{\tau}=(\tau, \phi)$, on définit l'indice de Souriau $m(\widetilde{\sigma}, \widetilde{\tau})$ par la formule

$$
m(\widetilde{\sigma}, \widetilde{\tau})=\frac{1}{\pi}[\Psi(\sigma, \tau)-r(\theta-\phi)] .
$$

On remarque tout de suite que $m$ est antisymétrique,

$$
m(\widetilde{\sigma}, \widetilde{\tau})+m(\widetilde{\tau}, \widetilde{\sigma})=0 .
$$

De plus. d'après (16)

$$
e^{2 i \pi m(\widetilde{\sigma}, \widetilde{\tau})}=e^{-2 i r \theta} e^{2 i r \phi}(\operatorname{det} \sigma)^{2}(\operatorname{det} \tau)^{-2}=1,
$$


de sorte que $m(\widetilde{\sigma}, \widetilde{\tau}) \in \mathbb{Z}$.

Enfin la fonction $m$ est une fonction continue (donc localement constante) sur $\widetilde{S}_{\text {T }}^{2}$, d'après la continuité de la fonction $\Psi$.

Proposition 5.4. La fonction $m$ est invariante sous l'action du groupe $\Gamma$.

Démonstration. Soit $\mathcal{U}$ un voisinage assez petit et simplement connexe de l'élément neutre de $G$. On peut trouver une détermination $\varphi(g, z)$ de l'argument de $j(g, z)$ qui soit définie sur $\mathcal{U} \times \overline{\mathcal{D}}$ et dépendant continûment du couple $(g, z)$. Soient $\widetilde{\sigma}=$ $(\sigma, \theta), \widetilde{\tau}=(\tau, \phi)$ deux éléments de $\widetilde{S}$, et supposons $\sigma \top \tau$. Soit $g \in \mathcal{U}$, alors $g(\sigma) \top g(\tau)$, et on a

$m((g, \varphi(g, \cdot))(\sigma, \theta),(g, \varphi(g, \cdot))(\tau, \phi))=\frac{1}{\pi}[\Psi(g(\sigma), g(\tau))-r(\theta-\phi)-(\varphi(g, \sigma)-\varphi(g, \tau))]$ ce qui montre que $g \longmapsto m((g, \varphi(g, \cdot))(\sigma, \theta),(g, \varphi(g, \cdot))(\tau, \phi))$ est une fonction continue. Comme elle est à valeurs dans $\mathbb{Z}$ elle est donc constante sur $\mathcal{U}$. Par ailleurs on vérifie facilement, d'après (8), que $m(T \cdot \widetilde{\sigma}, T \cdot \widetilde{\tau})=m(\widetilde{\sigma}, \widetilde{\tau})$. Le voisinage $\{(g, \varphi(g, \cdot) \mid g \in \mathcal{U}\}$ du neutre de $\Gamma$ et l'élément $T$ engendrent le groupe $\Gamma$, la fonction $m$ est bien invariante par $\Gamma$.

Soit maintenant

$$
S_{\top}^{3}=\left\{\left(\sigma_{1}, \sigma_{2}, \sigma_{3}\right) \in S^{3} \mid \sigma_{j} \top \sigma_{k}, 1 \leq j \neq k \leq 3\right\} .
$$

Fixons un repère de Jordan $\left(c_{j}\right)_{1 \leq j \leq r}$ et posons pour tout entier $k$ tel que $0 \leq k \leq r$

$$
\varepsilon_{0}=-e, \varepsilon_{k}=\sum_{j=1}^{k} c_{j}-\sum_{j=k+1}^{r} c_{j}, \varepsilon_{r}=e .
$$

Le groupe $G$ agit sur $S_{\top}^{3}$ et d'après $\left[\mathrm{C}-\varnothing_{1}\right.$, Theoreme 4.3$]$ cette action admet exactement $r+1$ orbites représentées par la famille $\left(e,-e,-i \varepsilon_{k}\right), 0 \leq k \leq r$.

Soit $\left(\sigma_{1}, \sigma_{2}, \sigma_{3}\right) \in S_{\text {个 }}^{3}$. L'indice de Maslov $\imath\left(\sigma_{1}, \sigma_{2}, \sigma_{3}\right)$ est défini dans $\left[\mathrm{C}-\emptyset_{1},(21)\right]$ par

$$
\imath\left(\sigma_{1}, \sigma_{2}, \sigma_{3}\right)=2 k-r
$$

où $k$ est l'unique entier tel que $\left(\sigma_{1}, \sigma_{2}, \sigma_{3}\right)$ soit conjugué à $\left(e,-e,-i \varepsilon_{k}\right)$.

Posons

$$
\widetilde{S}_{\top}^{3}=\left\{\left(\widetilde{\sigma}_{1}, \widetilde{\sigma}_{2}, \widetilde{\sigma}_{3}\right) \in S^{3} \mid \widetilde{\sigma}_{j} \top \widetilde{\sigma}_{k}, 1 \leq j \neq k \leq 3\right\} .
$$

Le théorème suivant montre que l'indice de Souriau est en fait une primitive de l'indice de Maslov.

Théorème 5.5 (Formule de Leray). Soit $\left(\widetilde{\sigma}_{1}, \widetilde{\sigma}_{2}, \widetilde{\sigma}_{3}\right) \in \widetilde{S}_{\top}^{3}$. Alors la quantité

$$
m\left(\widetilde{\sigma}_{1}, \tilde{\sigma}_{2}\right)+m\left(\widetilde{\sigma}_{2}, \widetilde{\sigma}_{3}\right)+m\left(\widetilde{\sigma}_{3}, \widetilde{\sigma}_{1}\right)
$$

ne dépend que des projections $\sigma_{1}, \sigma_{2}, \sigma_{3}$ et est égale à l'indice de Maslov $\imath\left(\sigma_{1}, \sigma_{2}, \sigma_{3}\right)$. Autrement dit

$$
m\left(\widetilde{\sigma}_{1}, \tilde{\sigma}_{2}\right)+m\left(\widetilde{\sigma}_{2}, \widetilde{\sigma}_{3}\right)+m\left(\widetilde{\sigma}_{3}, \widetilde{\sigma}_{1}\right)=\imath\left(\sigma_{1}, \sigma_{2}, \sigma_{3}\right) .
$$

Démonstration. Par un calcul direct, en utilisant (18), on montre que

$$
m\left(\widetilde{\sigma}_{1}, \widetilde{\sigma}_{2}\right)+m\left(\widetilde{\sigma}_{2}, \widetilde{\sigma}_{3}\right)+m\left(\widetilde{\sigma}_{3}, \widetilde{\sigma}_{1}\right)=\Psi\left(\sigma_{1}, \sigma_{2}\right)+\Psi\left(\sigma_{2}, \sigma_{3}\right)+\Psi\left(\sigma_{3}, \sigma_{1}\right) .
$$

Cette somme est donc un entier qui ne dépendent que des trois projections correspondantes. Soit $\jmath\left(\sigma_{1}, \sigma_{2}, \sigma_{3}\right)$ l'entier ainsi défini. C'est une fonction continue (donc 
en fait localement constante) de $S_{\top}^{3}$. Le groupe $G$ est connexe et opère continûment sur $S_{\text {个 }}^{3}$. Cela implique que la fonction $\jmath$ est invariante sous l'action de $G$. Pour montrer que $\jmath$ et $\imath$ cö̈ncident, il suffit donc de les comparer sur chaque classe de conjugaison de triplets sous l'action de $G$. Soit donc $k$ un entier tel que $0 \leq k \leq r$. On doit calculer $\jmath\left(e,-e,-i \varepsilon_{k}\right)$. On a

$$
e=\sum_{j=1}^{r} e^{i 0} c_{j}, \quad-e=\sum_{j=1}^{r} e^{i \pi} c_{j}, \quad-i \varepsilon_{k}=\sum_{j=1}^{k} e^{-i \frac{\pi}{2}} c_{j}+\sum_{j=k+1}^{r} e^{i \frac{\pi}{2}} c_{j},
$$

qu'on relève en les points

$$
\widetilde{e}=(e, 0), \widetilde{\widetilde{-e}}=(-e, \pi), \widetilde{\widetilde{-i} \varepsilon_{k}}=\left(-i \varepsilon_{k}, \frac{(r-2 k) \pi}{2 r}\right) .
$$

Un calcul simple en utilisant la formule (17) donne

$$
m(\widetilde{e}, \widetilde{-e})=r, \quad m\left(\widetilde{-e}, \widetilde{-i \varepsilon_{k}}\right)=-r, m\left(\widetilde{-i \varepsilon_{k}}, \widetilde{e}\right)=2 k-r .
$$

D'où

$$
m\left(\widetilde{\sigma}_{1}, \widetilde{\sigma}_{2}\right)+m\left(\widetilde{\sigma}_{2}, \widetilde{\sigma}_{3}\right)+m\left(\widetilde{\sigma}_{3}, \widetilde{\sigma}_{1}\right)=2 k-r=\imath\left(\sigma_{1}, \sigma_{2}, \sigma_{3}\right)
$$

\section{L'extension de L'indice de Souriau au CAS nOn transverse}

Dans $\left[\mathrm{C}_{2}\right]$ il est montré que l'indice de Maslov peut être défini pour tout triplet $\left(\sigma_{1}, \sigma_{2}, \sigma_{3}\right)$ de $S \times S \times S$. Cet indice, encore noté $\imath\left(\sigma_{1}, \sigma_{2}, \sigma_{3}\right)$ prolonge l'indice défini sur $S_{\frac{1}{3}}^{3}$ et possède encore les propriétés principales, à savoir l'invariance par le groupe $G$, l'antisymétrie et surtout la relation de cocycle que nous utiliserons dans la suite sous la forme

$$
\imath\left(\sigma_{1}, \sigma_{2}, \sigma_{3}\right)-\imath\left(\sigma_{1}, \sigma_{2}, \sigma_{4}\right)+\imath\left(\sigma_{1}, \sigma_{3}, \sigma_{4}\right)-\imath\left(\sigma_{2}, \sigma_{3}, \sigma_{4}\right)=0
$$

pour tous $\sigma_{1}, \sigma_{2}, \sigma_{3}, \sigma_{4} \in S$.

Il est donc naturel de chercher à étendre la définition de l'indice de Souriau aux couples non transverses pour obtenir une primitive de l'indice de Maslov étendu. Pour l'extension de la primitive $m$ aux couples non transverses, on va utiliser une idée due à de Gosson $\left[\mathrm{G}_{1}\right]$.

Montrons d'abord l'unicité de l'extension. Supposons que la fonction $m$ se prolonge à $\widetilde{S} \times \widetilde{S}$ en une primitive antisymétrique notée encore $m$ du cocycle de Maslov, c'est-à-dire telle que l'on ait pour tout $\left(\widetilde{\sigma}_{1}, \widetilde{\sigma}_{2}, \widetilde{\sigma}_{3}\right) \in \widetilde{S} \times \widetilde{S} \times \widetilde{S}$,

$$
\imath\left(\sigma_{1}, \sigma_{2}, \sigma_{3}\right)=m\left(\widetilde{\sigma}_{1}, \widetilde{\sigma}_{2}\right)+m\left(\widetilde{\sigma}_{2}, \widetilde{\sigma}_{3}\right)+m\left(\widetilde{\sigma}_{3}, \widetilde{\sigma}_{1}\right) .
$$

On en déduit que nécessairement

$$
m\left(\widetilde{\sigma}_{1}, \widetilde{\sigma}_{2}\right)=\imath\left(\sigma_{1}, \sigma_{2}, \sigma_{3}\right)+m\left(\widetilde{\sigma}_{1}, \widetilde{\sigma}_{3}\right)+m\left(\widetilde{\sigma}_{3}, \widetilde{\sigma}_{2}\right) .
$$

Étant donnés deux éléments $\widetilde{\sigma}_{1}, \widetilde{\sigma}_{2} \in \widetilde{S}$, on peut toujours trouver $\widetilde{\sigma}_{3} \in \widetilde{S}$ tels que $\tilde{\sigma}_{3} \top \widetilde{\sigma}_{1}$ et $\widetilde{\sigma}_{3} \top \widetilde{\sigma}_{2}$. Le membre de droite de l'égalité (20) est bien défini et il y a donc unicité de l'extension possible.

Théorème et définition 6.1. Soient $\widetilde{\sigma}_{1}, \widetilde{\sigma}_{2}$ deux éléments de $\widetilde{S}$ et soit $\widetilde{\tau}$ un élément quelconque de $\widetilde{S}$ tel que $\widetilde{\tau} \top \widetilde{\sigma}_{1}$ et $\widetilde{\tau} \top \widetilde{\sigma}_{2}$. Soient $\sigma_{1}, \sigma_{2}, \tau$ les projections respectives de $\widetilde{\sigma}_{1}, \widetilde{\sigma}_{2}, \widetilde{\tau}$. L'entier

$$
\imath\left(\sigma_{1}, \sigma_{2}, \tau\right)+m\left(\widetilde{\sigma}_{1}, \widetilde{\tau}\right)+m\left(\widetilde{\tau}, \widetilde{\sigma}_{2}\right)
$$


ne dépends pas du choix de $\widetilde{\tau}$ et est appelé l'indice de Souriau du couple $\left(\widetilde{\sigma}_{1}, \widetilde{\sigma}_{2}\right)$. Lorsque $\sigma_{1} \top \sigma_{2}$, il coïncide avec $m\left(\widetilde{\sigma}_{1}, \widetilde{\sigma}_{2}\right)$.

Démonstration. Soient $\widetilde{\sigma}_{1}, \widetilde{\sigma}_{2}$ deux éléments quelconques de $\widetilde{S}$. Alors on peut trouver deux éléments $\widetilde{\tau}_{1}, \widetilde{\tau}_{2}$ tels que

$$
\widetilde{\tau}_{1} \top \widetilde{\sigma}_{1}, \widetilde{\tau}_{1} \top \widetilde{\sigma}_{2}, \quad \widetilde{\tau}_{2} \top \widetilde{\sigma}_{1}, \widetilde{\tau}_{2} \top \widetilde{\sigma}_{2} \text {, et } \widetilde{\tau}_{1} \top \widetilde{\tau}_{2} \text {. }
$$

D'après le théorème 5.5 appliqué aux triplets transverses $\left(\widetilde{\sigma}_{1}, \widetilde{\tau}_{1}, \widetilde{\tau}_{2}\right)$ et $\left(\widetilde{\sigma}_{2}, \widetilde{\tau}_{1}, \widetilde{\tau}_{2}\right)$, on obtient

$$
\begin{aligned}
& \imath\left(\sigma_{1}, \tau_{1}, \tau_{2}\right)=m\left(\widetilde{\sigma}_{1}, \widetilde{\tau}_{1}\right)+m\left(\widetilde{\tau}_{1}, \widetilde{\tau}_{2}\right)+m\left(\widetilde{\tau}_{2}, \widetilde{\sigma}_{1}\right), \\
& \imath\left(\sigma_{2}, \tau_{1}, \tau_{2}\right)=m\left(\widetilde{\sigma}_{2}, \widetilde{\tau}_{1}\right)+m\left(\widetilde{\tau}_{1}, \widetilde{\tau}_{2}\right)+m\left(\widetilde{\tau}_{2}, \widetilde{\sigma}_{2}\right) .
\end{aligned}
$$

D'où

$$
\imath\left(\sigma_{1}, \tau_{1}, \tau_{2}\right)-\imath\left(\sigma_{2}, \tau_{1}, \tau_{2}\right)=m\left(\widetilde{\sigma}_{1}, \widetilde{\tau}_{1}\right)+m\left(\widetilde{\tau}_{2}, \widetilde{\sigma}_{1}\right)-m\left(\widetilde{\sigma}_{2}, \widetilde{\tau}_{1}\right)-m\left(\widetilde{\tau}_{2}, \widetilde{\sigma}_{2}\right) .
$$

En utilisant la relation de cocycle (19), cette dernière égalité devient

$$
\imath\left(\sigma_{1}, \sigma_{2}, \tau_{2}\right)+m\left(\widetilde{\sigma}_{1}, \widetilde{\tau}_{2}\right)+m\left(\widetilde{\tau}_{2}, \widetilde{\sigma}_{2}\right)=\imath\left(\sigma_{1}, \sigma_{2}, \tau_{1}\right)+m\left(\widetilde{\sigma}_{1}, \widetilde{\tau}_{1}\right)+m\left(\widetilde{\tau}_{1}, \widetilde{\sigma}_{2}\right) .
$$

D'où la première assertion du théorème.

Lorsque $\sigma_{1} \top \sigma_{2}$, la formule de Leray (théorème 5.5) appliquée au triplet $\left(\widetilde{\sigma}_{1}, \widetilde{\sigma}_{2}, \widetilde{\tau}\right)$ montre que $m\left(\widetilde{\sigma}_{1}, \widetilde{\sigma}_{2}\right)=\imath\left(\sigma_{1}, \sigma_{2}, \tau\right)+m\left(\widetilde{\sigma}_{1}, \widetilde{\tau}\right)+m\left(\widetilde{\tau}, \widetilde{\sigma}_{2}\right)$. D'où la deuxième assertion du théorème.

Soient $\widetilde{\sigma}_{1}, \widetilde{\sigma}_{2}$ deux éléments de $\widetilde{S}$ de projections respectives $\sigma_{1}$ et $\sigma_{2}$. On pose dans la suite

$$
m\left(\widetilde{\sigma}_{1}, \widetilde{\sigma}_{2}\right)=\imath\left(\sigma_{1}, \sigma_{2}, \tau\right)+m\left(\widetilde{\sigma}_{1}, \widetilde{\tau}\right)+m\left(\widetilde{\tau}, \widetilde{\sigma}_{2}\right),
$$

où $\widetilde{\tau} \in \widetilde{S}$ est un élément dont la projection $\tau$ est transverse à $\sigma_{1}$ et $\sigma_{2}$. Cette quantité est encore appelée l'indice de Souriau du couple $\left(\widetilde{\sigma}_{1}, \widetilde{\sigma}_{2}\right)$.

Remarque 6.2. On vérifie facilement les assertions suivantes:

(i) l'extension $m$ est antisymétrique.

(ii) l'extension $m$ est invariante sous l'action du groupe $\Gamma$.

Théorème 6.3 (Formule de Leray étendue). Soient $\left.\widetilde{\sigma}_{1}, \widetilde{\sigma}_{2}, \widetilde{\sigma}_{3}\right) \in \widetilde{S}$ de projections respectives $\sigma_{1}, \sigma_{2}, \sigma_{3}$, alors

$$
m\left(\widetilde{\sigma}_{1}, \widetilde{\sigma}_{2}\right)+m\left(\widetilde{\sigma}_{2}, \widetilde{\sigma}_{3}\right)+m\left(\widetilde{\sigma}_{3}, \widetilde{\sigma}_{1}\right)=\imath\left(\sigma_{1}, \sigma_{2}, \sigma_{3}\right) .
$$

Démonstration. Choisissons un élément $\widetilde{\tau} \in \widetilde{S}$ tel que $\widetilde{\tau}\rceil \widetilde{\sigma}_{1}, \widetilde{\tau} \top \widetilde{\sigma}_{2}$ et $\left.\widetilde{\tau}\right\rceil \widetilde{\sigma}_{3}$. Alors d'après la définition (21) de l'indice $m$ étendu on a

$$
\begin{aligned}
& m\left(\widetilde{\sigma}_{1}, \widetilde{\sigma}_{3}\right)=\imath\left(\sigma_{1}, \sigma_{3}, \tau\right)+m\left(\widetilde{\sigma}_{1}, \widetilde{\tau}\right)+m\left(\widetilde{\tau}, \widetilde{\sigma}_{3}\right), \\
& m\left(\widetilde{\sigma}_{2}, \widetilde{\sigma}_{3}\right)=\imath\left(\sigma_{2}, \sigma_{3}, \tau\right)+m\left(\widetilde{\sigma}_{2}, \widetilde{\tau}\right)+m\left(\widetilde{\tau}, \widetilde{\sigma}_{3}\right) .
\end{aligned}
$$

En utilisant encore une fois la relation de cocycle (19), on obtient

$$
\imath\left(\sigma_{1}, \sigma_{2}, \tau\right)+m\left(\widetilde{\sigma}_{1}, \widetilde{\tau}\right)+m\left(\widetilde{\tau}, \widetilde{\sigma}_{2}\right)=\imath\left(\sigma_{1}, \sigma_{2}, \sigma_{3}\right)+m\left(\widetilde{\sigma}_{1}, \widetilde{\sigma}_{3}\right)+m\left(\widetilde{\sigma}_{3}, \widetilde{\sigma}_{2}\right) .
$$

Le membre de gauche de cette dernière égalité étant par définition $m\left(\widetilde{\sigma}_{1}, \widetilde{\sigma}_{2}\right)$, le théorème s'en déduit en utilisant l'antisymétrie de l'indice de Souriau étendu. 
On va maintenant donner l'expression "en coordonnées" de l'indice de Souriau. Nous aurons besoin d'une notation particulière, qui n'est en fait qu'une version de l'indice de Maslov pour le cas du cercle unité.

Soient donnés $e^{i \theta}, e^{i \phi}$ deux nombres complexes de module 1 distincts. On notes ]$e^{i \theta}, e^{i \phi}\left[\right.$ l'arc de cercle d'extrémités $e^{i \theta}$ et $e^{i \phi}$ formé des points obtenus en partant de $e^{i \theta}$ dans le sens trigonométrique pour arriver à $e^{i \phi}$.

Pour $e^{i \theta}, e^{i \varphi}$ et $e^{i \phi}$ trois points du cercle orienté, on pose

$$
\operatorname{ord}\left(e^{i \theta}, e^{i \varphi}, e^{i \phi}\right)=\left\{\begin{aligned}
0 & \text { si deux des points } e^{i \theta}, e^{i \varphi}, e^{i \phi} \text { coïncident, } \\
1 & \text { si } \left.e^{i \varphi} \in\right] e^{i \theta}, e^{i \phi}[ \\
-1 & \text { si } \left.e^{i \varphi} \in\right] e^{i \phi}, e^{i \theta}[
\end{aligned}\right.
$$

Proposition 6.4. Soient $\sigma_{1}=\sum_{j=1}^{r} e^{i \theta_{j}} c_{j}, \sigma_{2}=\sum_{j=1}^{r} e^{i \varphi_{j}} c_{j}$ et $\sigma_{3}=\sum_{j=1}^{r} e^{i \phi_{j}} c_{j}$ trois points de $S$. Alors

$$
\imath\left(\sigma_{1}, \sigma_{2}, \sigma_{3}\right)=\sum_{j=1}^{r} \operatorname{ord}\left(e^{i \theta_{j}}, e^{i \varphi_{j}}, e^{i \phi_{j}}\right) .
$$

Démonstration. Cette formule est implicitement contenue dans $\left[\mathrm{C}_{2}\right]$.

Fixons maintenant un repère de Jordan $\left(c_{j}\right)_{1 \leq j \leq r}$.

Proposition 6.5. Soient

$$
\tilde{\sigma}_{1}=\left(\sum_{j=1}^{\ell} e^{i \theta_{j}} c_{j}+\sum_{j=\ell+1}^{r} e^{i \theta_{j}} c_{j}, \theta\right), \quad \widetilde{\sigma}_{2}=\left(\sum_{j=1}^{\ell} e^{i \theta_{j}} c_{j}+\sum_{j=\ell+1}^{r} e^{i \varphi_{j}} c_{j}, \varphi\right),
$$

deux éléments tels que $\theta_{j}-\varphi_{j} \notin 2 \pi \mathbb{Z}$ pour $\ell+1 \leq j \leq r$. Alors

$$
m\left(\widetilde{\sigma}_{1}, \widetilde{\sigma}_{2}\right)=\frac{1}{\pi}\left[\sum_{j=\ell+1}^{r}\left\{\theta_{j}-\varphi_{j}+\pi\right\}-r(\theta-\varphi)\right] .
$$

Démonstration. Pour un réel assez petit $\phi>0$, l'élément

$$
\tilde{\sigma}_{3}=\left(\sum_{j=1}^{\ell} e^{i\left(\theta_{j}+\phi\right)} c_{j}+\sum_{j=\ell+1}^{r} e^{i\left(\varphi_{j}+\phi\right)} c_{j}, \varphi+\phi\right),
$$

est transverse à $\widetilde{\sigma}_{1}$ et à $\widetilde{\sigma}_{2}$. D'une part, en appliquant la formule (23), l'indice de Maslov des projections $\sigma_{1}, \sigma_{2}$ et $\sigma_{3}$ est

$$
\imath\left(\sigma_{1}, \sigma_{2}, \sigma_{3}\right)=r-\ell .
$$

D'autre part, en utilisant (17) et (18), on obtient

$$
\begin{aligned}
m\left(\widetilde{\sigma}_{1}, \widetilde{\sigma}_{3}\right) & =\frac{1}{\pi}\left[\left(\sum_{j=1}^{\ell}\{-\phi+\pi\}+\sum_{j=\ell+1}^{r}\left\{\theta_{j}-\varphi_{j}-\phi+\pi\right\}\right)-r(\theta-(\varphi+\phi))\right], \\
& =\ell+\frac{1}{\pi}\left[\sum_{j=\ell+1}^{r}\left\{\theta_{j}-\varphi_{j}+\pi\right\}-r(\theta-\varphi)\right], \\
\text { et } & \begin{aligned}
m\left(\widetilde{\sigma}_{3}, \widetilde{\sigma}_{2}\right) & =\frac{1}{\pi}\left[\left(\sum_{j=1}^{\ell}\{\phi+\pi\}+\sum_{j=\ell+1}^{r}\{\phi+\pi\}\right)-r \phi\right], \\
& =-r .
\end{aligned}
\end{aligned}
$$


Finalement, d'après (21), l'indice de Souriau $m\left(\widetilde{\sigma}_{1}, \widetilde{\sigma}_{2}\right)$ est donné par

$$
m\left(\widetilde{\sigma}_{1}, \widetilde{\sigma}_{2}\right)=\frac{1}{\pi}\left[\sum_{j=\ell+1}^{r}\left\{\theta_{j}-\varphi_{j}+\pi\right\}-r(\theta-\varphi)\right] .
$$

En particulier si $\widetilde{\sigma}_{1}=(-e,-\pi)$ et $\widetilde{\sigma}_{2}=\left(-\sum_{j=1}^{\ell} c_{j}+\sum_{j=\ell+1}^{r} e^{i \varphi_{j}} c_{j}, \varphi\right)$, où

(i) $-\pi<\varphi_{j}<\pi, \forall j, \ell+1 \leq j \leq r$, et alors

(ii) $r \varphi=-\ell \pi+\sum_{j=\ell+1}^{r} \varphi_{j}+2 k \pi$, avec $k \in \mathbb{Z}$,

$$
m\left(\widetilde{\sigma}_{1}, \widetilde{\sigma}_{2}\right)=2 k+r-\ell=2 k+r-\mu\left(\sigma_{1}, \sigma_{2}\right) .
$$

Remarque 6.6. Pour $\widetilde{\sigma}, \widetilde{\tau} \in \widetilde{S}$, on a

$$
m(\widetilde{\sigma}, T \cdot \widetilde{\tau})=-m(T \cdot \widetilde{\sigma}, \widetilde{\tau})=m(\widetilde{\sigma}, \widetilde{\tau})+2
$$

\section{Le CyCle De Maslov}

Soit $\sigma_{0} \in S$. On note $\Sigma\left(\sigma_{0}\right)$ l'ensemble des éléments de $S$ qui ne sont pas transverses à $\sigma_{0}$, c'est-à-dire

$$
\Sigma\left(\sigma_{0}\right)=S \backslash S_{\top}\left(\sigma_{0}\right)=\left\{\sigma \in S \mid \operatorname{det}\left(\sigma-\sigma_{0}\right)=0\right\} .
$$

Cet ensemble est appelé le cycle de Maslov associé à $\sigma_{0}$.

On va, dans ce paragraphe étudier sa structure de variété stratifiée (au sens algébrique).

7.1. La variété des éléments de $J$ de corang $k$. Reprenons les notations du paragraphe 4 .

Proposition 7.1. Soit $c$ un idempotent, et $J=J(c, 1) \oplus J\left(c, \frac{1}{2}\right) \oplus J(c, 0)$ la décomposition de Peirce relative à cet idempotent. L'application donnée par

$$
J(c, 1) \times J\left(c, \frac{1}{2}\right) \times J(c, 0) \rightarrow J:\left(x_{1}, x_{\frac{1}{2}}, x_{0}\right) \mapsto \tau_{c}\left(x_{\frac{1}{2}}\right)\left(x_{1}+x_{0}\right)
$$

est un difféomorphisme local près de tout point $\left(x_{1}^{\circ}, x_{\frac{1}{2}}^{\circ}, x_{0}^{\circ}\right)$ tel que $x_{1}^{\circ}$ soit inversible comme élément de $J(c, 1)$.

Démonstration. Soit $\Phi$ la représentation de l'algèbre de Jordan $J(c, 1)$ dans $\operatorname{End}\left(J\left(c, \frac{1}{2}\right)\right)$ donnée par (13). D'après les formules (11) on a, en posant, $\tau_{c}\left(x_{\frac{1}{2}}\right)\left(x_{1}+x_{0}\right)=$ $y_{1}+y_{\frac{1}{2}}+y_{0}$

$$
\begin{aligned}
& y_{1}=x_{1}, \\
& y_{\frac{1}{2}}=\Phi\left(x_{1}\right) x_{\frac{1}{2}}, \\
& y_{0}=2 L(e-c) L\left(x_{\frac{1}{2}}\right)^{2} x_{1}+x_{0} .
\end{aligned}
$$

Si l'élément $x_{1}^{\circ}$ considéré comme élément de la sous-algèbre $J(c, 1)$ est inversible, l'endomorphisme $\Phi\left(x_{1}^{\circ}\right)$ est inversible. Ceci reste vrai pour $x_{1}$ voisin de $x_{1}^{\circ}$. Il en résulte facilement que l'application considérée est un difféomorphisme local près de $\left(x_{1}^{\circ}, x_{\frac{1}{2}}^{\circ}, x_{0}^{\circ}\right)$.

Un élément $x$ de $J$ est dit de corang $k$ si son rang est $r-k$. Pour tout entier $k$ tel que $0 \leq k \leq r$, on note dans la suite $J_{k}$ l'ensemble des éléments de $J$ de corang $k$,

$$
J_{k}=\{x \in J \mid \mu(x, 0)=k\} .
$$


Ainsi, $J_{0}$ n'est autre que l'ensemble $J^{\times}$des éléments inversibles de $J$, et $J_{r}=\{0\}$.

Proposition 7.2. Soit $k$ un entier tel que $1 \leq k \leq r-1$. Alors $J_{k}$ est une sousvariété de $J$ de codimension $k+\frac{k(k-1)}{2} d$. En particulier, Si $k=1$, alors $J_{1}$ est de codimension 1 , et si $k \geq 2$, alors la codimension de $J_{k}$ est supérieur ou égale à 3 .

Démonstration. Soit $1 \leq k \leq r-1$. Soit $x^{\circ} \in J_{k}$ un élément de rang $r-k$. Il existe donc une décomposition de Peirce $e=\sum_{j=1}^{r} c_{j}$ telle que

$$
x^{\circ}=\sum_{j=1}^{r-k} \lambda_{j} c_{j}
$$

avec $\lambda_{j} \neq 0$ pour tout $j, 1 \leq j \leq r-k$. Posons $c=\sum_{j=1}^{r-k} c_{j}$. L'élément $c$ ainsi défini est un idempotent de l'algèbre de Jordan $J$. Soit

$$
J=J(c, 1) \oplus J\left(c, \frac{1}{2}\right) \oplus J(c, 0)
$$

la décomposition de Peirce de $J$ relative à cet idempotent. L'élément $x^{\circ}$ appartient à $J(c, 1)$ et est un élément inversible de $J(c, 1)$. Par suite, on peut appliquer la proposition 7.1. On obtient ainsi des coordonnées locales $\left(y_{1}(x), y_{\frac{1}{2}}(x), y_{0}(x)\right)$ près de $\left(x^{\circ}, 0,0\right)$, telles que $x=\tau_{c}\left(y_{\frac{1}{2}}(x)\right)\left(y_{1}(x)+y_{0}(x)\right)$. L'action du groupe $L$ préservant la notion de rang d'un élément, le rang de $x$ est le même que le rang de $y_{1}(x)+y_{0}(x)$. Mais le rang de $y_{1}+y_{0}$ est la somme du rang de l'élément $y_{1}$ et de l'élément $y_{0}$, pour tout $y_{1} \in J(c, 1), y_{0} \in J(c, 0)$. Pour $x$ voisin de $x^{\circ}, y_{1}(x)$ est voisin de $x^{0}$ et donc inversible dans l'algèbre de Jordan $J(c, 1)$, ou si l'on préfère de rang $r-k$. Par conséquent la condition

$$
y_{0}(x)=0
$$

fournit un système d'équations locales de $J_{k}$ près de $x^{\circ}$. Ceci montre que $J_{k}$ est une sous-variété de codimension égale à $\operatorname{dim} J(c, 0)$. D'après (10) cette dimension est

$$
\operatorname{dim} J(c, 0)=k+\frac{k(k-1)}{2} d,
$$

puisque l'idempotent $c$ est de rang $r-k$.

Si $k=1$ alors $\operatorname{dim} J(c, 0)=1$ et $J_{1}$ est de codimension 1 .

Si $k \geq 2$, alors $\operatorname{dim} J(c, 0) \geq 3$, puisque l'invariant de Peirce $d$ d'une algèbre de Jordan euclidienne simple est non nul (voir [F-K, page 70]), et donc la codimension de $J_{k}$ est supérieure ou égale à 3 .

D'après [F-K, Proposition II.4.2], le gradient de la fonction det est polynomial et vérifie sur $J^{\times}$la formule

$$
\nabla \operatorname{det}(x)=\operatorname{det}(x) x^{-1} .
$$

Plus explicitement, si $x=\sum_{j=1}^{r} \lambda_{j} c_{j}$ est une décomposition de Peirce d'un élément inversible de $J$, alors

$$
\nabla \operatorname{det}(x)=\sum_{j=1}^{r}\left(\lambda_{1} \cdots \stackrel{\vee}{\lambda}_{j} \cdots \lambda_{r}\right) c_{j} .
$$

L'ensemble $J \backslash J^{\times}=\sqcup_{k=1}^{r} J_{k}$ est l'ensemble des éléments $x \in J$ tels que $\operatorname{det}(x)=0$. C'est une variété (au sens algébrique) de codimension 1, dont on va maintenant préciser le lieu singulier. 
Proposition 7.3. Soit $x \in \sqcup_{k=1}^{r} J_{k}$. Alors, $\nabla \operatorname{det}(x) \neq 0$ si, et seulement si, $x \in J_{1}$.

Démonstration. Soit $x \in J_{1}$, alors il existe un repère de Jordan $\left(c_{j}\right)_{1 \leq j \leq r}$ et des nombres réels non nuls $\left(\lambda_{j}\right)_{1 \leq j \leq r-1}$ tels que $x=\sum_{j=1}^{r-1} \lambda_{j} c_{j}$. On pose $x_{\epsilon}=$ $\sum_{j=1}^{r-1} \lambda_{j} c_{j}+\epsilon c_{r}$ pour $\epsilon>0$ assez petit. D'après la formule (28) on a

$$
\nabla \operatorname{det}\left(x_{\epsilon}\right)=\sum_{j=1}^{r-1} \epsilon\left(\lambda_{1} \cdots \stackrel{\vee}{\lambda_{j}} \cdots \lambda_{r-1}\right) c_{j}+\left(\lambda_{1} \cdots \lambda_{r-1}\right) c_{r} .
$$

Par conséquent, lorsque $\epsilon \rightarrow 0, \nabla \operatorname{det}\left(x_{\epsilon}\right) \rightarrow \lambda_{1} \cdots \lambda_{r-1} c_{r}$. Par continuité, on a donc

$$
\nabla \operatorname{det}(x)=\left(\lambda_{1} \cdots \lambda_{r-1}\right) c_{r} \neq 0 .
$$

Si par contre $x \in \sqcup_{k=2}^{r} J_{k}$, un calcul similaire montre que $\nabla \operatorname{det}(x)=0$. D'où la proposition.

7.2. La stratification du cycle de Maslov. Étant donné un élément $\sigma_{0} \in S$, et $k$ un entier, $0 \leq k \leq r$, on pose

$$
S_{k}\left(\sigma_{0}\right)=\left\{\sigma \in S \mid \mu\left(\sigma, \sigma_{0}\right)=k\right\} .
$$

On a

$$
\Sigma\left(\sigma_{0}\right)=\bigsqcup_{1 \leq k \leq r} S_{k}\left(\sigma_{0}\right) .
$$

Dans le cas où $S$ est la variété lagrangienne $\Lambda(r)$, pour un sous-espace lagrangien $\lambda_{0}$ fixé, $S_{k}\left(\lambda_{0}\right)$ coïncide avec l'ensemble $\Lambda^{k}(r)$ des sous-espaces lagrangiens $\lambda$ tels que $\operatorname{dim}\left(\lambda \cap \lambda_{0}\right)=k$.

Proposition 7.4. $S_{k}\left(\sigma_{0}\right)$ est une sous-variété de $S$ de codimension $k+\frac{k(k-1)}{2} d$.

Démonstration. Soit $\sigma \in S_{k}\left(\sigma_{0}\right)$. Grâce à l'action du groupe $G$, on peut toujours supposer que $\sigma_{0}=-e$, et $\sigma \top e$. Par la transformation de Cayley, on est ramené à l'énoncé équivalent pour $J_{k}$, qui a été démontré précédemment (proposition 7.2).

Proposition 7.5. Le cycle de Maslov $\Sigma\left(\sigma_{0}\right)$ est une sous-variété stratifiée de $S$ (au sens algébrique) de codimension 1. Le lieu singulier de $\Sigma\left(\sigma_{0}\right)$ est de codimension supérieure ou égale à 3 dans $S$.

Démonstration. Utilisant comme précédemment la transformation de Cayley, on voir d'abord, grâce à la proposition 7.3 que $S_{1}\left(\sigma_{0}\right)$ est l'ensemble des points réguliers de $\Sigma\left(\sigma_{0}\right)$, et $S_{1}\left(\sigma_{0}\right)$ est de codimension 1 dans $S$. Le lieu singulier est égal à $\sqcup_{2 \leq k \leq r} S_{k}\left(\sigma_{0}\right)$, qui est de codimension $\geq 3$ dans $S$, d'après la proposition 7.2.

7.3. L'orientation transverse du cycle de Maslov. Dans le cas de la variété lagrangienne $\Lambda(r)$ (voir $\left[\mathrm{A}_{1}\right]$ ), Arnold montre que le cycle de Maslov admet un champ continu de vecteurs tangents à $\Lambda(r)$ et transverses à $\Lambda^{1}(r)$. Un tel champs de vecteurs est constitué par les vecteurs $v(\sigma)=\frac{d}{d \theta}\left(e^{i \theta} \sigma\right)_{\left.\right|_{\theta=0}}$ pour $\sigma \in \Lambda^{1}(r)$ et défini une orientation transverse. Nous allons montrer comment cette notion se généralise à $S$ en utilisant sa structure causale. 
Proposition 7.6. Soit $\sigma_{0} \in S$, et soit $\sigma \in S_{1}\left(\sigma_{0}\right) \subset \Sigma\left(\sigma_{0}\right)$. Soit $H_{\sigma_{0}}(\sigma)$ l'espace tangent à $\Sigma\left(\sigma_{0}\right)$ au point $\sigma$. Les vecteurs tangents aux courbes causales partant de $\sigma$ sont tous contenus dans un même demi-espace ouvert de l'espace tangent à $S$ en $\sigma$ limité par $H_{\sigma_{0}}(\sigma)$.

Par la transformée de Cayley, on est ramené à l'énoncé équivalent suivant.

Proposition 7.7. Soit $x \in J_{1} \subset J$. Soit $H(x)$ l'hyperplan tangent à $J_{1}$ en $x$. Les vecteurs tangents aux courbes causales partant de $x$ sont tous contenus dans un même demi-espace ouvert limité par $H(x)$.

Démonstration. On peut supposer que $x=\sum_{j=1}^{r-1} \lambda_{j} c_{j}$, où $\left(c_{j}\right)_{1 \leq j \leq r}$ est un repère de Jordan de $J$ et, pour $1 \leq j \leq r-1$, les $\lambda_{j}$ sont des scalaires non nuls. On sait alors qu'un vecteur normal (non nul!) est donné par

$$
\nabla \operatorname{det}(x)=\left(\prod_{j=1}^{r-1} \lambda_{j}\right) c_{r} .
$$

Si $y(t)$ est une courbe causale telle que $y(0)=x$ et $\dot{y}(0)=\omega \in \Omega$, on a donc

$$
\frac{d}{d t}(\operatorname{det} y(t))_{\left.\right|_{t=0}}=\langle\dot{y}(0) \mid \nabla \operatorname{det} x\rangle=\left(\prod_{j=1}^{r-1} \lambda_{j}\right)\left\langle\omega \mid c_{r}\right\rangle .
$$

Comme $\omega \in \Omega$ et $c_{r} \in \bar{\Omega} \backslash\{0\}$, on a $\left\langle\omega \mid c_{r}\right\rangle>0$, et par suite le signe de $\langle\dot{y}(0) \mid \nabla \operatorname{det} x\rangle$ ne dépend pas de la courbe causale choisie.

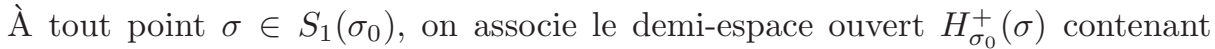
les vecteurs tangents aux courbes causales passant par $\sigma$. La famille de ces sousespaces définit une orientation transverse canonique du cycle de Maslov $\Sigma\left(\sigma_{0}\right)$. De plus ces orientations transverses sont compatibles à l'action de $G$, en ce sens que si $g \in G$, alors l'orientation transverse canonique du cycle de Maslov $\Sigma\left(g \sigma_{0}\right)$ au point $g \sigma \in S_{1}\left(g \sigma_{0}\right)$ est donnée par le demi-espace $D g(\sigma)\left(H_{\sigma_{0}}^{+}(\sigma)\right)=H_{g \sigma_{0}}^{+}(g \sigma)$, puisque la notion de courbe causale est stable par l'action de $G$.

\section{Le nombre D'ARnold, L'INDICE D'INERTIE ET L'INDICE D'ARNOLD-LERAY-MASLOV}

Les principales propriétés topologiques des cycles de Maslov démontrées dans le paragraphe précédent généralisent celles montrées par Arnold dans le cas de la variété lagrangienne $\Lambda(r)$. Dans ce cas particulier, Arnold (voir $\left[\mathrm{A}_{1}\right]$ et $\left[\mathrm{A}_{2}\right]$ ) utilise ces propriétés pour construire un indice pour les couples de points transverses du revêtement universel $\widetilde{\Lambda(r)}$. Les arguments d'Arnold sont encore valables dans notre situation et nous permettent dans la suite de proposer une deuxième construction, géométrique cette fois-ci, d'une primitive.

Soit $\sigma_{0} \in S$, et soit $\gamma$ un chemin dans $S$, d'origine $\gamma(0)=\sigma$, et d'extrémité $\gamma(1)=$ $\tau$. Un tel chemin est dit admissible (relativement au cycle de Maslov $\Sigma_{0}=\Sigma\left(\sigma_{0}\right)$ ) s'il est de classe $\mathcal{C}^{1}$ par morceaux, si ses extrémités $\sigma$ et $\tau$ n'appartiennent pas à $\Sigma_{0}$, si le chemin n'intersecte le cycle de Maslov que pour un nombre fini de valeurs de $t \in[0,1]$, (disons $\left.t=t_{1}, t_{2}, \ldots, t_{k}\right)$, si chaque point d'intersection $\gamma\left(t_{j}\right)$ est un point régulier de $\Sigma_{0}$ (c'est-à-dire $\gamma\left(t_{j}\right) \in S_{1}\left(\sigma_{0}\right)$ ), et enfin si en un tel point, la courbe $\gamma$ est différentiable et transverse à $\Sigma_{0}$. Le nombre d'Arnold d'un tel chemin admissible 
est par définition la somme $\epsilon_{1}+\epsilon_{2}+\cdots+\epsilon_{k}$, où $\epsilon_{j}$ vaut +1 si $\dot{\gamma}\left(t_{j}\right) \in H_{\sigma_{0}}^{+}\left(\gamma\left(t_{j}\right)\right)$, et $\epsilon_{j}$ vaut -1 dans le cas contraire.

Théorème 8.1. Soient $\sigma$ et $\tau$ deux points n'appartenant pas au cycle de Maslov $\Sigma_{0}$. Toute classe d'homotopie de chemins d'origine $\sigma$ et d'extrémité $\tau$ contient un chemin admissible. Deux chemins admissibles d'origine $\sigma$ et d'extrémité $\tau$ qui sont homotopes ont même nombre d'Arnold.

La démonstration est identique au cas classique (voir $\left[\mathrm{A}_{2}\right]$ ou $[\mathrm{C}-\mathrm{L}-\mathrm{M}]$ ), au vu des résultats du paragraphe 7 .

Ce théorème permet d'étendre la notion de nombre d'Arnold à un chemin quelconque $\gamma$ d'origine $\sigma$ et d'extrémité $\tau$, en le déclarant égal par définition à l'indice d'un chemin admissible de mêmes origine et extrémité et homotope à $\gamma$.

On va maintenant tirer profit de l'invariance par homotopie du nombre d'Arnold pour définir l'indice d'Arnold d'un couple de points du revêtement universel $\widetilde{S}$.

Soient $\widetilde{\sigma}_{0}$ et $\widetilde{\tau}_{0}$ deux points de $\widetilde{S}$, de projections respectives $\sigma_{0}$ et $\tau_{0}$ sur $S$. Soit $\sigma(t), 0 \leq t \leq 1$ une courbe causale d'origine $\sigma_{0}$, et $\tau(t), 0 \leq t \leq 1$ une courbe anti-causale d'origine $\tau_{0}$. On note $\widetilde{\sigma(t)}$ (resp. $\left.\widetilde{\tau(t)}\right)$ la courbe dans $\widetilde{S}$ d'origine $\widetilde{\sigma_{0}}$ (resp. $\widetilde{\tau_{0}}$ ) relevant $\sigma(t)$ (resp. $\tau(t)$ ). D'après la proposition 4.20 , il existe $\epsilon>0$ tel que pour tout $t, 0<t<\epsilon$, les points $\sigma(t)$ et $\tau(t)$ n'appartiennent pas au cycle de Maslov $\Sigma_{0}=\Sigma\left(\sigma_{0}\right)$. Fixons un tel $t$, et soit $\gamma_{t}(s), 0 \leq s \leq 1$ un chemin admissible (relativement à $\Sigma_{0}$ ) d'origine $\sigma(t)$ et d'extrémité $\tau(t)$, dont le relevé dans $\widetilde{S}$ a pour origine $\widetilde{\sigma}(t)$ et pour extrémité $\widetilde{\tau}(t)$.

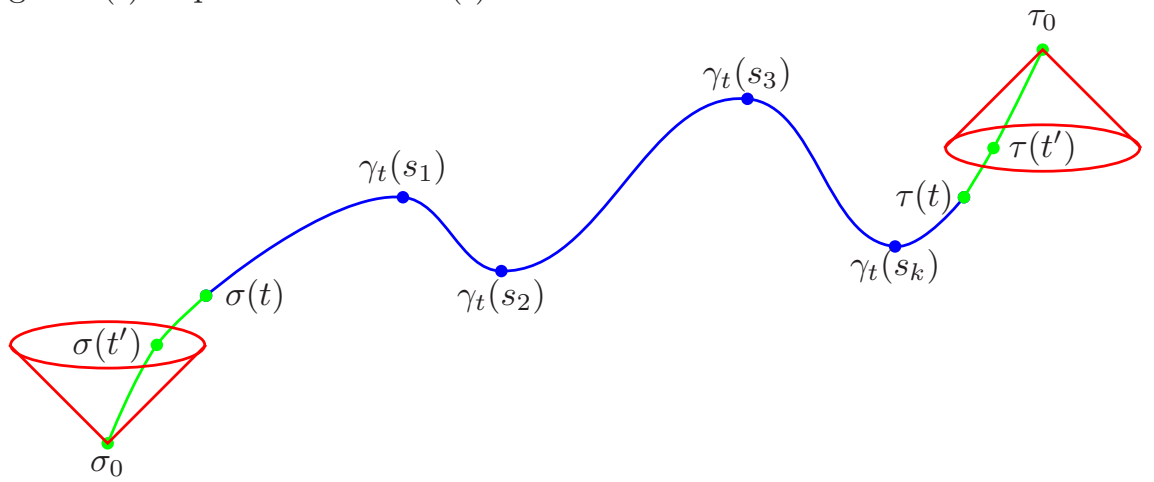

Fig. 1

Par définition l'indice d'Arnold $\nu\left(\widetilde{\sigma}_{0}, \widetilde{\tau}_{0}\right)$ est le nombre d'Arnold du chemin $\gamma_{t}$. Clairement, à $t$ fixé, cet indice ne dépend pas du choix du chemin $\gamma_{t}$, puisque tout autre chemin ayant les mêmes propriétés lui est homotope et donc a même nombre d'Arnold.

De plus, l'indice est indépendant du paramètre $t$ (voir Fig 1). En effet, soit $t^{\prime}$ une autre valeur, avec toujours $0<t^{\prime}<\epsilon$. On peut toujours supposer que $t^{\prime}<t$. On considère le chemin d'origine $\sigma\left(t^{\prime}\right)$ et d'extrémité $\tau\left(t^{\prime}\right)$ obtenu en concaténant le chemin $\left(\sigma(u), t^{\prime} \leq u \leq t\right)$, le chemin $\gamma_{t}$ de $\sigma(t)$ à $\tau(t)$, et enfin le chemin $(\tau(v)$, $\left.t^{\prime} \leq v \leq t\right)$. Il est immédiat que le nombre d'Arnold de ce chemin concaténé est le même que le nombre d'Arnold du chemin $\gamma_{t}$, puisque les deux bouts de chemin 
concaténés à $\gamma_{t}$ ne rencontrent pas le cycle de Maslov $\Sigma_{0}$, comme on le déduit aisément de la proposition 4.9.

Enfin, cet indice est indépendant des courbes (causale ou anti-causale) $\sigma(t)$ et $\tau(t)$. En effet, étant donné deux courbes causales $\sigma_{1}(t), 0 \leq t \leq 1$ et $\sigma_{2}(t), 0 \leq t \leq 1$ de même origine $\sigma_{0}$, on peut pour $t$ assez petit, joindre $\sigma_{1}(t)$ à $\sigma_{2}(t)$ par une courbe qui ne rencontre pas le cycle de Maslov $\Sigma_{0}$, comme on le voit en utilisant la convexité du cône $\Omega$.

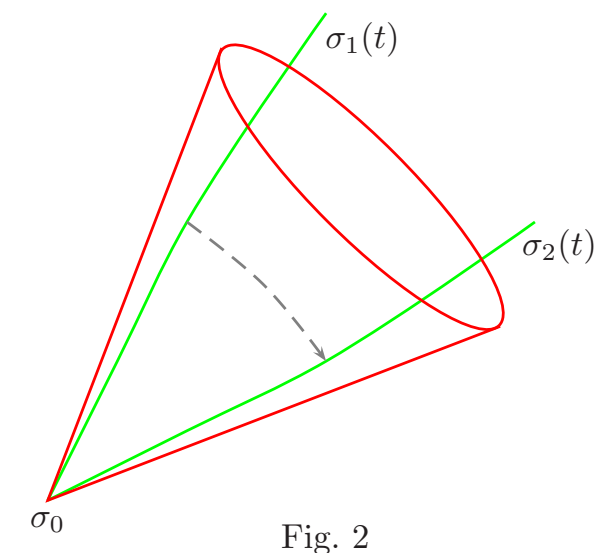

Un argument analogue vaut pour la courbe anti-causale d'origine $\tau_{0}$. Notons que si $\tau_{0}$ est transverse à $\sigma_{0}$, il n'est pas nécessaire d'utiliser une telle courbe anticausale, et on peut supposer que le chemin $\gamma_{t}$ est d'extrémité $\tau_{0}$.

La construction de l'indice d'Arnold ne fait appel qu'à des notions invariantes par transformations causales (courbes causales, cycle de Maslov, transversalité), et donc l'indice d'Arnold est invariant par l'action de $\Gamma$.

On va maintenant calculer l'indice d'Arnold "en coordonnées", comme nous l'avons fait pour l'indice de Souriau. On fixe pour cela un repère de Jordan $\left(c_{j}\right)_{1 \leq j \leq r}$ de $J$. Grâce aux résultats concernant les orbites de l'action de $G$ dans $S \times S$ (voir paragraphe 4), la proposition suivante couvre le cas général.

Proposition 8.2. Soient les deux points de $\widetilde{S}$,

$$
\widetilde{\sigma}_{0}=\widetilde{-e}=(-e,-\pi) \text { et } \widetilde{\tau}_{0}=\left(-\sum_{j=1}^{\ell} c_{j}+\sum_{j=\ell+1}^{r} e^{i \varphi_{j}} c_{j}, \varphi\right) .
$$

On note $\sigma_{0}=-e$ et $\tau_{0}$ leurs projections correspondantes dans $S$. On suppose que

(i) $-\pi<\varphi_{j}<\pi, \forall j, \ell+1 \leq j \leq r$;

(ii) $r \varphi=-\ell \pi+\sum_{j=\ell+1}^{r} \varphi_{j}+2 k \pi$, avec $k \in \mathbb{Z}$.

Alors

$$
\nu\left(\widetilde{\sigma}_{0}, \widetilde{\tau}_{0}\right)=-\ell+k=k-\mu\left(\sigma_{0}, \tau_{0}\right) .
$$

Démonstration. Le point $\sigma=\sum_{j=1}^{r} e^{i \theta_{j}} c_{j}$ appartient au cycle de Maslov $\Sigma_{0}=$ $\Sigma(-e)$ si et seulement s'il existe $j, 1 \leq j \leq r$ tel que $\theta_{j} \in-\pi+2 \pi \mathbb{Z}$. Il est régulier (c'est-à-dire $\sigma \in S_{1}(-e)$ ) si et seulement si $\theta_{j} \in-\pi+2 \pi \mathbb{Z}$ pour une seule 
valeur de l'indice $j$. Si, par exemple, $\sigma=-c_{1}+\sum_{j=2}^{r} e^{i \theta_{j}} c_{j}$, avec pour $2 \leq j \leq r$, $\theta_{j} \notin-\pi+2 \pi \mathbb{Z}$, alors un vecteur normal au plan tangent en $\sigma$ à $\Sigma_{0}$ donnant l'orientation positive est le vecteur $-i c_{1}$.

Comme courbe causale d'origine $\sigma_{0}$, choisissons la courbe

$$
\sigma(t)=\sum_{j=1}^{r} e^{i(-\pi+t)} c_{j}
$$

dont le relevé dans $\widetilde{S}$ partant de $\widetilde{\sigma}_{0}$ est

$$
\widetilde{\sigma(t)}=\left(\sum_{j=1}^{r} e^{i(-\pi+t)} c_{j},-\pi+t\right)
$$

et pour courbe anti-causale d'origine $\widetilde{\tau}_{0}$

$$
\tau(t)=\sum_{j=1}^{\ell} e^{i(-\pi-t)} c_{j}+\sum_{j=\ell+1}^{r} e^{i \varphi_{j}} c_{j}
$$

dont le relevé dans $\widetilde{S}$ est

$$
\widetilde{\tau(t)}=\left(\tau(t), \frac{1}{r}\left[\ell(-\pi-t)+\sum_{j=\ell+1}^{r} \varphi_{j}+2 k \pi\right]\right) .
$$

On notera que la courbe $\tau(t)$ n'est pas, au sens strict, anti-causale, puisque son vecteur tangent en chaque point est seulement dans la frontière du cône causal. Néanmoins, il est vrai que pour $t>0$ assez petit, le point $\tau(t)$ n'est pas dans $\Sigma_{0}$, et permet donc le calcul de l'indice d'Arnold du couple $\left(\widetilde{\sigma}_{0}, \widetilde{\tau}_{0}\right)$.

Ayant fixé un tel $t>0$ assez petit, le chemin $\gamma_{t}$ va être obtenu par concaténation de plusieurs morceaux de chemins admissibles, dont on va calculer pour chacun la contribution à l'indice de d'Arnold. Pour la commodité de la lecture, chaque morceau de chemin est paramétré par $s \in[0,1]$.

Le premier morceau de chemin est donné par

$$
\gamma_{t}(s)=e^{i[(1-s)(-\pi+t)+s(-\pi-t)]} c_{1}+\sum_{j=2}^{r} e^{i s(-\pi+t)} c_{j} .
$$

Pour $s=\frac{1}{2}, \gamma_{t}(s)=-c_{1}+\sum_{j=2}^{r} e^{i t(-\pi+t)} c_{j}$, et c'est le seul point d'intersection du chemin $\gamma_{t}$ avec $\Sigma_{0}$. De plus, $\dot{\gamma}_{t}\left(\frac{1}{2}\right)=2 i t c_{1}$, de sorte que le chemin $\gamma_{t}$ coupe donc transversalement le cycle de Maslov, et la contribution à l'indice est -1 .

On continue par le même type de chemin, pour chaque $j, 2 \leq j \leq \ell$. L'extrémité finale de ces chemins est alors le point

$$
\sigma_{t}^{\prime}=\sum_{j=1}^{\ell} e^{i(-\pi-t)} c_{j}+\sum_{j=\ell+1}^{r} e^{i(-\pi+t)} c_{j}
$$

et la contribution à l'indice d'Arnold est $-\ell$. 
On considère maintenant le chemin

$$
\gamma_{t}(s)=\sum_{j=1}^{\ell} e^{i(-\pi-t)} c_{j}+\sum_{j=\ell+1}^{r} e^{\left((1-s)(-\pi+t)+s \varphi_{j}\right)} c_{j} .
$$

Ce chemin ne rencontre pas le cycle de Maslov $\Sigma_{0}$, et donc la contribution de ce morceau de chemin à l'indice est nulle. Le point d'arrivée est maintenant

$$
\tau_{t}^{\prime}=\sum_{j=1}^{\ell} e^{i(-\pi-t)} c_{j}+\sum_{j=\ell+1}^{r} e^{i \varphi_{j}} c_{j} .
$$

Un calcul facile permet de déterminer l'extrémité du relevé dans $\widetilde{S}$ du chemin parcouru jusqu'à présent. On trouve que c'est le point

$$
\widetilde{\tau_{t}^{\prime}}=\left(\tau_{t}^{\prime}, \frac{1}{r}\left[\ell(-\pi-t)+\sum_{j=\ell+1}^{r} \varphi_{j}\right]\right) .
$$

Pour terminer, on a besoin du lemme suivant.

Lemme 8.3. Soient donnés $\left(\psi_{j}\right)_{1 \leq j \leq r} r$ nombres réels tels que $\psi_{j} \notin-\pi+2 \pi \mathbb{Z}$. Soit $\gamma$ le chemin défini pour $0 \leq s \leq 1$ par

$$
\gamma(s)=e^{i\left(\psi_{1}+2 s \pi\right)} c_{1}+\sum_{j=2}^{r} e^{i \psi_{j}} c_{j}
$$

Le chemin $\gamma$ est admissible relativement au cycle de Maslov $\Sigma_{0}$, et son nombre d'Arnold vaut +1 .

En effet, le chemin $\gamma$ ne coupe $\Sigma_{0}$ que pour une seule valeur de $s$, transversalement, et dans le sens positif. Donc le nombre d'Arnold de ce chemin vaut +1 .

Ce lemme permet de calculer l'indice d'Arnold de deux points de $\widetilde{S}$ ayant même projection dans $S$. En particulier, en passant du point $\widetilde{\tau_{t}^{\prime}}$ au point $\widetilde{\tau_{t}}$, la contribution à l'indice d'Arnold est $k$. D'où la proposition 8.2.

Corollaire 8.4. Soient $\widetilde{\sigma}, \widetilde{\tau}$ deux éléments de $\widetilde{S}$ de projections respectives $\sigma$ et $\tau$. Alors

$$
\nu(\widetilde{\sigma}, \widetilde{\tau})=\frac{1}{2}(m(\widetilde{\sigma}, \widetilde{\tau})-\mu(\sigma, \tau)-r) .
$$

Démonstration. Il suffit en effet de comparer les formules (26) et (29) et d'utiliser l'invariance des deux membres par $\Gamma$.

Remarque 8.5. Un sous-produit de ce corollaire est que le membre de droite de la formule (30) est un entier (et non un demi-entier). On tire profit de ce résultat en introduisant la notion d'indice d'inertie et d'indice d'Arnold-Leray-Maslov.

Definition 8.6. (i) Soient $\left(\sigma_{1}, \sigma_{2}, \sigma_{3}\right) \in S^{3}$. On appelle indice d'inertie $d u$ triplet $\left(\sigma_{1}, \sigma_{2}, \sigma_{3}\right)$ la quantité

$$
\jmath\left(\sigma_{1}, \sigma_{2}, \sigma_{3}\right)=\frac{1}{2}\left(\iota\left(\sigma_{1}, \sigma_{2}, \sigma_{3}\right)+\mu\left(\sigma_{1}, \sigma_{2}\right)-\mu\left(\sigma_{1}, \sigma_{3}\right)+\mu\left(\sigma_{2}, \sigma_{3}\right)+r\right) .
$$


(ii) Soient $\widetilde{\sigma}_{1}, \widetilde{\sigma}_{2} \in \widetilde{S}$, de projections respectives $\sigma_{1}, \sigma_{2}$. On appelle indice d'ArnoldLeray-Maslov la quantité

$$
n\left(\widetilde{\sigma}_{1}, \widetilde{\sigma}_{2}\right)=\nu\left(\widetilde{\sigma}_{1}, \widetilde{\sigma}_{2}\right)+\mu\left(\sigma_{1}, \sigma_{2}\right)+r .
$$

Théorème 8.7. L'indice d'inertie satisfait les propriétés suivantes :

(i) J est à valeurs entières.

(ii) J vérifie la relation de 2-cocycle ${ }^{4}$

$$
\jmath\left(\sigma_{1}, \sigma_{2}, \sigma_{3}\right)-\jmath\left(\sigma_{1}, \sigma_{2}, \sigma_{4}\right)+\jmath\left(\sigma_{1}, \sigma_{3}, \sigma_{4}\right)-\jmath\left(\sigma_{2}, \sigma_{3}, \sigma_{4}\right)=0
$$

pour tous $\sigma_{1}, \sigma_{2}, \sigma_{3} \in S$.

(iii) l'indice d'Arnold-Leray-Maslov est une primitive de l'indice d'inertie, c'està-dire

$$
\jmath\left(\sigma_{1}, \sigma_{2}, \sigma_{3}\right)=n\left(\widetilde{\sigma}_{1}, \widetilde{\sigma}_{2}\right)-n\left(\widetilde{\sigma}_{1}, \widetilde{\sigma}_{3}\right)+n\left(\widetilde{\sigma}_{2}, \widetilde{\sigma}_{3}\right)
$$

pour tous $\widetilde{\sigma}_{1}, \widetilde{\sigma}_{2}, \widetilde{\sigma}_{3} \in \widetilde{S}$, de projections respectives $\sigma_{1}, \sigma_{2}, \sigma_{3}$.

Démonstration. ( $i)$ et $($ ii $)$ sont des conséquences faciles de $($ iii $)$. Pour $($ iii $)$, on utilise la relation

$$
n(\widetilde{\sigma}, \widetilde{\tau})=\frac{1}{2}(m(\widetilde{\sigma}, \widetilde{\tau})+\mu(\sigma, \tau)+r)
$$

valable pour tous $\widetilde{\sigma}, \widetilde{\tau} \in \widetilde{S}$ de projections respectives $\sigma, \tau$, qui est conséquence du corollaire 8.4. On voit alors que la relation ( iii) est équivalente à la propriété de cocycle pour l'indice de Maslov (19).

\section{L'Indice DE MASLOV D'Une PAIRE DE ChEMins DANS $S \times S$}

Dans ce paragraphe, nous allons adapter une méthode due à Cappell-Lee-Miller [C-L-M] pour expliquer (sans plus de détails) comment on peut définir l'indice de Maslov d'une paire de chemins de $S$.

Un chemin $[0,1] \ni t \mapsto \varpi(t)=\left(\sigma_{1}(t), \sigma_{2}(t)\right) \in S \times S$ est propre, si $\sigma_{1}(t)$ est transverse à $\sigma_{2}(t)$ pour $t=0$ et $t=1$.

Soit $\varpi$ un tel chemin et supposons qu'il est régulier. D'après le paragraphe 7 , si on note

$$
\mathcal{Z}=\left\{(t, \sigma) \in[-1,1] \times S, \operatorname{det}\left(\sigma_{1}(t)-\sigma\right)=0\right\}
$$

alors $\mathcal{Z} \cap\{t\} \times S=\{t\} \times \Sigma\left(\sigma_{1}(t)\right)$ est sous-variété stratifiée (au sens algébrique), transversalement orientée, de codimension 1 de $\{t\} \times S$ et dont la singularité est de codimension $\geq 3$ dans $\{t\} \times S$. Cette singularité est donc de codimension $\geq 2$ dans $\mathcal{Z}$. Par conséquent moyennant une petite perturbation du chemin $\varpi$, tout en gardant ses extrémités fixes, on peut transformer le chemin $t \mapsto\left(t, \sigma_{2}(t)\right)$ en un autre chemin $t \mapsto\left(t, \sigma_{2}^{\prime}(t)\right)$ ne rencontrant $\mathcal{Z}$ qu'en un nombre fini de points situés sur la strate supérieure $\{t\} \times S_{1}\left(\sigma_{1}(t)\right)$ et ceci d'une manière transverse.

Definition 9.1. L'indice de Maslov $\nu_{p}(\varpi)$ du chemin propre $\varpi$ est par définition le nombre d'intersections, comptés avec leurs signes, du chemin $t \mapsto\left(t, \sigma_{2}^{\prime}(t)\right)$ avec $\mathcal{Z}$.

${ }^{4}$ On observera que l'indice d'inertie $\jmath$ n'a pas la propriété d'antisymétrie que possède l'indice de Maslov $\imath$. 
La réunion disjointe de toutes les strates supérieures

$$
\bigcup_{0<t<1}\{t\} \times S_{1}\left(\sigma_{1}(t)\right)
$$

est une sous-variété ouverte de $\mathcal{Z}$ dont la singularité est de codimension supérieure ou égale à 3 dans $\mathcal{Z}$. On en déduit que deux petites perturbations du chemin $\left(\cdot, \sigma_{2}^{\prime}(\cdot)\right)$ sont homotopes et par conséquent $\nu_{p}(\varpi)$ est indépendant du choix de cette perturbation.

Remarquons enfin que si le chemin $\varpi$ est seulement continue, on peut l'approcher par un chemin régulier et définir son indice $\nu_{p}(\varpi)$. Comme la singularité est de codimension $\geq 3$, l'indice $\nu_{p}(\varpi)$ est indépendant du choix de cette approximation.

Les deux propositions suivantes sont une conséquence directe de la définition de l'indice $\nu_{p}$.

Proposition 9.2. Soit $\varpi:[0,1] \rightarrow S \times S, t \mapsto\left(\sigma_{1}(t), \sigma_{2}(t)\right)$ un chemin propre et régulier.

(1) Si $\sigma_{1}(t)$ est transverse à $\sigma_{2}(t)$ pour tout $t \in[0,1]$, alors $\nu_{p}(\varpi)=0$.

(2) $S i[0,1] \ni t \mapsto \hat{\varpi}(t)=\left(\sigma_{1}(-t), \sigma_{2}(-t)\right)$ est le chemin inverse de chemin $\varpi$, alors $\nu_{p}(\varpi)=\nu_{p}(\hat{\varpi})$.

(3) Pour tout $\alpha \in] 0,1[$,

$$
\nu_{p}(\varpi)=\nu_{p}\left(\varpi_{[0, \alpha]}\right)+\nu_{p}\left(\varpi_{\left.\right|_{[\alpha, 1]}}\right)
$$

(4) Soit $g=\left(g_{t}\right)_{0 \leq t \leq 1}$ une famille d'éléments de $G$ telle que $[0,1] \ni t \mapsto g_{t}$ soit continue. Si on pose $(g * \varpi)(t)=\left(g_{t}\left(\sigma_{1}(t)\right), g_{t}\left(\sigma_{2}(t)\right)\right.$ pour $t \in[0,1]$, alors

$$
\nu_{p}(g * \varpi)=\nu_{p}(\varpi)
$$

Proposition 9.3. Si $[a, b] \times[0,1] \ni(s, t) \mapsto\left(\sigma_{1}(s, t), \sigma_{2}(s, t)\right) \in S \times S$ est une application continue, et si pour tout $s \in[a, b]$, le chemin $\varpi_{s}(t)=\left(\sigma_{1}(s, t), \sigma_{2}(s, t)\right)$ est propre et régulier dans $S \times S$, alors

$$
\nu_{p}\left(\varpi_{0}\right)=\nu_{p}\left(\varpi_{1}\right)
$$

La fonction $f: \theta \mapsto \operatorname{det}\left(\sigma_{1}(t)-e^{i \theta} \sigma_{2}(t)\right)$ a un nombre fini de zéros (modulo $2 \pi$ ). Il existe donc $\epsilon \in] 0, \pi$ [ tels que les $\theta$ qui vérifient $0<|\theta|<\pi$ ne soient pas des zéros de $f$. D'où le lemme suivant :

Lemme 9.4. Soit $\varpi:[0,1] \rightarrow S \times S, t \mapsto\left(\sigma_{1}(t), \sigma_{2}(t)\right)$ un chemin régulier dans $S \times S$. Alors il existe $\epsilon \in] 0, \pi[$ tel que pour tout $\theta$ vérifiant $0<|\theta|<\epsilon$, le nouveau chemin perturbé

$$
\varpi_{\theta}(t)=\left(\sigma_{1}(t), e^{i \theta} \sigma_{2}(t)\right)
$$

soit propre.

La proposition suivante se déduit facilement de la proposition 9.3 et du lemme 9.4 . 
Proposition 9.5. Soit $\varpi$ un chemin régulier dans $S \times S$. Si $\varpi_{\theta_{1}}$ et $\varpi_{\theta_{2}}$ sont deux perturbations différentes de $\varpi$ en chemins propres, alors

$$
\nu_{p}\left(\varpi_{\theta_{1}}\right)=\nu_{p}\left(\varpi_{\theta_{2}}\right)
$$

Definition 9.6. Soit $\varpi$ un chemin régulier dans $S \times S$. L'indice de Maslov de $\varpi$ est par définition l'indice de Maslov d'une perturbation $\varpi_{\theta}$ de $\varpi$ :

$$
\nu(\varpi)=\nu_{p}\left(\varpi_{\theta}\right) .
$$

La proposition 9.5 montre que l'indice $\nu(\varpi)$ est bien défini et ne dépend pas de la perturbation choisie.

Finalement les propositions 9.2 et 9.3 s'étendent à l'indice de Maslov $\nu(\varpi)$ d'un chemin quelconque $\varpi$. Pour plus de détails nous invitons le lecteur à consulter le travail de Cappell-Lee-Miller $[\mathrm{C}-\mathrm{L}-\mathrm{M}]$ dans lequel cette théorie est très bien développée.

\section{LE NOMBRE DE ROTATION GÉNÉRALISÉ}

Dans $[B-G]$, les auteurs montrent qu'on peut utiliser une primitive (quelconque) du cocycle classique de Maslov pour construire un invariant (pour la conjugaison) sur le groupe symplectique, baptisé nombre de rotation symplectique. Nous montrons qu'une construction analogue est possible dans la situation considérée dans ce travail.

Commençons par quelques lemmes à caractère général.

Lemme 10.1. Soit $G$ un groupe de Lie semi-simple à centre fini et connexe. Soit $G=K A N$ une décomposition d'Iwasawa de $G$, et soit $r=\operatorname{dim} A$ le rang déployé de G. Alors

(i) tout élément de $N$ est un commutateur,

(ii) tout élément de A peut s'écrire comme produit d'au plus $r$ commutateurs.

Démonstration. $(i)$ Soit $\mathfrak{a}=\operatorname{Lie}(A), H$ un élément régulier de $\mathfrak{a}$ et $h=\exp H$. Alors l'application

$$
n \mapsto h n h^{-1} n^{-1}, \quad N \rightarrow N
$$

est un difféomorphisme de $N$ sur lui-même (voir $\left[\mathrm{He}_{1}, \mathrm{Ch}\right.$. VI. Corollary 4.8]).

(ii) Soit $\Sigma$ le système de racines restreintes relativement à a et $\Sigma^{+}$l'ensemble des racines positives tel que

$$
\operatorname{Lie}(N)=\mathfrak{n}=\bigoplus_{\alpha \in \Sigma^{+}} \mathfrak{g}_{\alpha} .
$$

Soit $\left\{\alpha_{1}, \alpha_{2}, \ldots, \alpha_{r}\right\}$ les racines simples correspondantes. Pour $j, 1 \leq j \leq r$, on note $H_{j}$ le covecteur associé à la racine $\alpha_{j}$. La symétrie orthogonale associée à la racine $\alpha_{j}$ est un élément du groupe de Weyl, et on en choisit un représentant $m_{j}$ dans le normalisateur de $\mathfrak{a}$ dans $K$. On a alors, pour tout $j, 1 \leq j \leq r$

$$
m_{j} \exp H_{j} m_{j}^{-1}=\exp \left(-H_{j}\right) \text {. }
$$

Maintenant, soit $h=\exp H$ un élément quelconque de $A$. On a

$$
H=\sum_{j=1}^{r} t_{j} H_{j}, \quad h=\prod_{j=1}^{r} \exp t_{j} H_{j}, \quad t_{j} \in \mathbb{R}, 1 \leq j \leq r .
$$


D'après (31)

$$
\exp t_{j} H_{j}=m_{j} \exp \left(-\frac{t_{j}}{2} H_{j}\right) m_{j}^{-1} \exp \left(\frac{t_{j}}{2} H_{j}\right)
$$

est donc un commutateur, et l'assertion (ii) en découle.

Lemme 10.2. Soit

$$
0 \longrightarrow \mathbb{Z} \stackrel{\imath}{\longrightarrow} \Gamma \stackrel{p}{\longrightarrow} G \longrightarrow 1
$$

une extension centrale d'un groupe de Lie G semi-simple à centre fini et connexe. Notons $T=\imath(1)$. Il existe au plus une application $\Phi: \Gamma \longrightarrow \mathbb{R}$ telle que

(i) $\Phi$ est continue

(ii) $\Phi(\gamma T)=\Phi(\gamma)+1, \forall \gamma \in \Gamma$

(iii) $\Phi\left(\gamma_{1} \gamma_{2}\right)-\Phi\left(\gamma_{1}\right)-\Phi\left(\gamma_{2}\right)$ est bornée sur $\Gamma \times \Gamma$

(iv) $\Phi\left(\gamma^{n}\right)=n \Phi(\gamma), \forall \gamma \in \Gamma, \forall n \in \mathbb{Z}$.

Démonstration. Soit $\Phi_{1}$ et $\Phi_{2}$ deux telles fonctions, D'après (ii), la fonction $\Phi_{1}-\Phi_{2}$ passe au quotient et définit une fonction $F$ sur $G$. D'après ( $(i i i)$ elle est uniformément bornée sur les commutateurs, et donc sur $N$ et sur $A$. D'après $(i)$ elle est bornée sur $K$. Utilisant la décomposition d'Iwasawa et à nouveau (iii), on voit que $F$ est uniformément bornée sur $G$. D'après $(i v)$, cela implique que $F$ est identiquement nulle. D'où $\Phi_{1}=\Phi_{2}$.

Une telle fonction $\Phi$ est appelée un quasi-morphisme homogène ${ }^{5}$. Un quasimorphisme définit, par passage au quotient, une application $\Psi: G \longrightarrow \mathbb{R} / \mathbb{Z}$.

Revenant aux notations du paragraphe 5 , on va maintenant construire un quasimorphisme pour le groupe $\Gamma$.

Soit $\widetilde{o}$ un point base dans $\widetilde{S}$, et posons pour $\gamma \in \Gamma$

$$
c(\gamma)=m(\gamma \cdot \widetilde{o}, \widetilde{o}) .
$$

La primitive $m(\widetilde{\sigma}, \widetilde{\tau})$ est invariante par $\Gamma$, et donc pour tous $\gamma_{1}, \gamma_{2} \in \Gamma$

$$
\begin{aligned}
c\left(\gamma_{1} \gamma_{2}\right)-c\left(\gamma_{1}\right)-c\left(\gamma_{2}\right) & =m\left(\gamma_{1} \gamma_{2} \cdot \widetilde{o}, \widetilde{o}\right)-m\left(\gamma_{1} \cdot \widetilde{o}, \widetilde{o}\right)-m\left(\gamma_{2} \cdot \widetilde{o}, \widetilde{o}\right) \\
& =m\left(\gamma_{1} \gamma_{2} \cdot \widetilde{o}, \widetilde{o}\right)+m\left(\widetilde{o}, \gamma_{1} \cdot \widetilde{o}\right)+m\left(\gamma_{1} \cdot \widetilde{o}, \gamma_{1} \gamma_{2} \cdot \widetilde{o}\right)
\end{aligned}
$$

qui d'après (22) est l'indice de Maslov des projections sur $S$ des points $\gamma_{1} \gamma_{2} \cdot \widetilde{o}, \widetilde{o}$ et $\gamma_{1} \cdot \widetilde{o}$ de sorte que

$$
\left|c\left(\gamma_{1} \gamma_{2}\right)-c\left(\gamma_{1}\right)-c\left(\gamma_{2}\right)\right| \leq r .
$$

Pour tout élément $\gamma \in \Gamma$, la suite $\left(c_{k}\right):=\left(c\left(\gamma^{k}\right)\right)_{k \in \mathbb{N}}$ satisfait donc la propriété

$$
\forall k, \ell \in \mathbb{N}, \quad\left|c_{k+\ell}-c_{k}-c_{\ell}\right| \leq r .
$$

D'après un résultat classique, on déduit que

$$
\tau(\gamma)=\lim _{k \rightarrow+\infty} \frac{1}{k} c\left(\gamma^{k}\right)
$$

existe pour tout élément $\gamma \in \Gamma$.

Théorème 10.3. La fonction

$$
-\frac{1}{2} \tau
$$

est un quasi-morphisme sur $\Gamma$. Elle est indépendante du choix de $\widetilde{o}$.

\footnotetext{
${ }^{5}$ Notre définition diffère légèrement de celle de $[\mathrm{B}-\mathrm{G}]$.
} 
Démonstration. La continuité de $\tau$ se démontre comme dans [B-G, Proposition 2.11]. Les autres propriétés sont des conséquences faciles des propriétés de la primitive $m$ et notamment (27). L'indépendance du point base résulte du lemme 10.2, compte-tenu de ce que le groupe $G$ est semi-simple, à centre fini et connexe.

Par passage au quotient le quasi-morphisme $-\frac{1}{2} \tau$ défini une fonction de $G$ dans $\mathbb{R} / \mathbb{Z}$ par

$$
\rho(g)=-\frac{1}{2} \tau(\gamma) \quad \bmod \mathbb{Z}
$$

où $\gamma$ est un relèvement quelconque de $g$. Pour $g \in G$, la quantité $\rho(g)$ est appelée nombre de rotation généralisé de $g$. La fonction $\rho$ est invariante par conjugaison (toujours d'après l'unicité).

Proposition 10.4. La fonction $\rho$ possède les propriétés suivantes :

(i) si $g \in G$ fixe un point de $S$, alors $\rho(g)=0 \bmod \mathbb{Z}$.

(ii) si $u \in U$, alors $e^{2 i \pi \rho(u)}=\chi(u)$.

Démonstration. Pour $(i)$, on choisit comme origine $\widetilde{o}$ dans $\widetilde{S}$ un point qui se projette sur un point de $S$ fixé par $g$, et on relève $g$ en un élément $\gamma$ tel que $\gamma \cdot \widetilde{o}=\widetilde{o}$, et donc aussi $\gamma^{k} \cdot \widetilde{o}=\widetilde{o}$ pour tout $k \in \mathbb{N}$. Il en résulte que $\tau(\gamma)=0$.

Pour $(i i)$, choisissons un réel $\varphi$ tel que $\chi(u)=e^{i r \varphi}$. Un relèvement de l'élément $u$ est l'élément $\gamma$ dont l'action sur $\widetilde{S}$ est définie par

$$
\gamma \cdot \widetilde{\sigma}=\gamma \cdot(\sigma, \theta)=(u(\sigma), \theta+\varphi),
$$

puisqu'en effet $\operatorname{det}(u(\sigma))=\chi(u) \operatorname{det}(\sigma)=e^{i r \varphi} e^{i r \theta}=e^{i r(\theta+\varphi)}$. Donc

$$
\frac{1}{k} c\left(\gamma^{k}\right)=\frac{1}{k} m\left(\gamma^{k} \cdot \widetilde{\sigma}, \widetilde{\sigma}\right)=\frac{1}{k} \frac{1}{\pi}\left(\Psi\left(u^{k}(\sigma), \sigma\right)-k r \varphi\right) \rightarrow-\frac{\varphi r}{\pi}
$$

quand $k \rightarrow+\infty$, puisque $\Psi$ est une fonction bornée. Par suite,

$$
\rho(u)=\frac{\varphi r}{2 \pi}
$$

et donc $e^{2 i \pi \rho(u)}=e^{i r \varphi}=\chi(u)$.

\section{RÉFÉRENCES}

[A 1 Arnol'd, V. I. On a characteristic class entering into conditions of quantization. Funkcional. Anal. i Prilozen. 1 (1967) 1-14.

$\left[\mathrm{A}_{2}\right]$ Arnol'd, V. I. Sturm theorems and symplectic geometry. Funktsional. Anal. i Prilozhen. 19 (1985), 1-10.

[B-G] Barge J.; Ghys E. Cocycles d'Euler et de Maslov, Math. Ann. 294 (1991), 235-265.

[B] Bertram, W. Un théorème de Liouville pour les algèbres de Jordan. Bull. Soc. Math. France 124 (1996), 299-327.

[C-L-M] Cappell, S. E.; Lee, R.; Miller, E. Y. On the Maslov index. Comm. Pure Appl. Math. 47 (1994), 121-186.

[C- $\left.\varnothing_{1}\right]$ Clerc, J. L.; Ørsted, B. The Maslov index revisited. Transform. Groups 6 (2001), 303-320.

$\left[\mathrm{C}-\varnothing_{2}\right]$ Clerc, J. L.; Ørsted, B. The Gromov norm of the Kaehler class and the Maslov index. Asian J. Math. 7 (2003), 269-296.

$\left[\mathrm{C}_{1}\right]$ Clerc, J. L. The Maslov index on the Shilov boundary of a classical domain. J. Geom. Physics 49 (2004), 21-51.

$\left[\mathrm{C}_{2}\right]$ Clerc, J. L. L'indice de Maslov généralisé. J. Math. Pures Appl. 83 (2004), 99-114. 
$\left[\mathrm{G}_{1}\right]$ de Gosson, M. La définition de l'indice de Maslov sans hypothèse de transversalité. C. $R$. Acad. Sci. Paris Sér. I Math. 310 (1990), 279-282.

$\left[\mathrm{G}_{2}\right]$ de Gosson, M. Maslov classes, metaplectic representation and Lagrangian quantization. Mathematical Research, 95. Akademie-Verlag, Berlin, 1997.

[F-K] Faraut, J.; Korányi, A. Analysis on Symmetric Cones, Oxford Mathematical Monographs, Clarendon Press, Oxford, 1994.

[God] Godement, R. Introduction à la théorie des groupes de Lie. Tome 1. Publications Mathématiques de l'Université Paris VII, 11, Université de Paris VII, U.E.R. de Mathématiques, Paris, 1982.

[G-S] Guillemin, V.; Sternberg, S. Geometric asymptotics. Mathematical Surveys, 14. American Mathematical Society, Providence, R.I., 1977.

$\left[\mathrm{He}_{1}\right]$ Helgason, S. Differential geometry and symmetric spaces. Pure and Applied Mathematics, Vol. XII. Academic Press, New York-London, 1962.

$\left[\mathrm{He}_{2}\right]$ Helgason, S. Differential geometry, Lie groups, and symmetric spaces. Pure and Applied Mathematics, 80. Academic Press, Inc. New York - London, 1978.

[Hu] Hua, L.K. Geometries of matrices. I. Generalizations of von Staudt's theorem. Trans. Amer. Math. Soc. 57, (1945), 441-481.

[Ka] Kaneyuki, S. On the causal structures of the Šilov boundaries of symmetric bounded domains. Prospects in complex geometry (Katata and Kyoto, 1989), 127-159, Lecture Notes in Math., 1468, Springer, Berlin, 1991.

[Ko] Koufany, K. Semi-groupe de Lie associé un cône symétrique. Ann. Inst. Fourier 45 (1995), $1-29$.

[Le] Leray, J. Analyse lagrangienne et mécanique quantique. Séminaire sur les Équations aux Dérivées Partielles (1976-1977), I, Exp. No. 1, 303 pp. Collège de France, Paris, 1977.

$[\mathrm{L}-\mathrm{V}]$ Lion, G.; Vergne, M. The Weil representation, Maslov index and theta series. Progress in Mathematics, 6. Birkhäuser, Boston, Mass., 1980.

[Lo] Loos, O. Symmetric spaces. II : Compact spaces and classification. W. A. Benjamin, Inc., New York-Amsterdam, 1969.

[M] Maslov, V. P. Théorie des perturbations et méthodes asymptotiques. Dunod, Paris, 1972. (avec annexe par Arnol'd, V.I.)

[S] Souriau, J. M. Construction explicite de l'indice de Maslov. Applications. Lecture Notes in Phys., Vol. 50, pp. 117-148. Springer, Berlin, 1976.

Institut Élie Cartan, UMR 7502, Université Henri Poincaré (Nancy 1) B.P. 239, 54506 VANDEUURE-LÈs-NANCY CEDEX, France

E-mail address: Jean-Louis.Clerc@iecn.u-nancy.fr, Khalid.Koufany@iecn.u-nancy.fr 